\title{
Asymptotic Shallow Models Arising in Magnetohydrodynamics
}

\section{Diego Alonso-Orán ${ }^{1}$}

Received: 25 September 2020 / Accepted: 27 April 2021 / Published online: 17 May 2021

(c) The Author(s) 2021

\begin{abstract}
In this paper, we derive new shallow asymptotic models for the free boundary plasmavacuum problem governed by the magnetohydrodynamic equations which are vital when describing large-scale processes in flows of astrophysical plasma. More precisely, we present the magnetic analogue of the 2D Green-Naghdi equations for water waves under a weak magnetic pressure assumption in the presence of weakly sheared vorticity and magnetic currents. Our method is inspired by ideas for hydrodynamic flows developed in Castro and Lannes (2014) to reduce the three-dimensional dynamics of the vorticity and current to a finite cascade of two dimensional equations which can be closed at the precision of the model.
\end{abstract}

Keywords MHD equations · Shallow approximation · Magnetic Green-Naghdi equations with shear

Mathematics Subject Classification 76W05 $\cdot 35 \mathrm{Q} 35 \cdot 35 \mathrm{R} 35 \cdot 34 \mathrm{E} 05$

\section{Introduction}

Plasma is an ionized gas consisting of freely moving positively charged ions, electrons and neutrals. It is by far the most common phase of ordinary matter present in the Universe. We are in constant contact with the small amounts that are not plasma, such as oceans and seas, but electrically charged fluids are everywhere throughout the galaxy [45]. Macroscopic plasma processes are usually described by the so-called magnetohydrodynamic equations (MHD), first proposed by the physicist Alfvén [2]. Here the behaviour of fluid particles is governed by Newton's second law under the effect of an electromagnetic force described by Maxwell's equations. Furthermore,

\footnotetext{
$凶$ Diego Alonso-Orán

alonso@iam.uni-bonn.de

1 Institute for Applied Mathematics, University of Bonn, Endenicher Allee 60, 53115 Bonn, Germany
} 
the movements of present charged particles creates an electric field that also affects the magnetic field.

Motivated by the problem of magnetic plasma confinement in laboratory research, the plasma-vacuum interface problem has attracted the interest of the mathematical community in the last decades $[21,22,42,43]$. However, studying the full dynamics of the equations is too complex, mainly because the moving surface boundary is part of the solution. This led physicists, oceanographers and mathematicians to derive and replace the original equations by approximate asymptotic systems in specific physical regimes. Those systems are more amenable to numerical simulations and their properties are more transparent.

In the case of the water waves equations describing the motion of an inviscid and incompressible fluid delimited by a free-surface, the most prominent asymptotic model are the non-linear shallow water equations, also known as Saint-Venant equations, see $[1,33]$. In the shallow water regime, when $\mu:=\frac{H_{0}^{2}}{L^{2}} \ll 1$ (where $L$ is the typical horizontal scale and $H_{0}$ the typical depth), the non-linear shallow water system is derived from the free-surface Euler equation by averaging and neglecting the $\mathcal{O}(\mu)$ order terms. As a consequence of simplifying the model by dropping the $\mathcal{O}(\mu)$ order terms dispersive effects are missing which are however crucial for many applications. Keeping them in the equations and neglecting the $\mathcal{O}\left(\mu^{2}\right)$ order terms, one obtains the so-called Green-Naghdi equations [18] or Serre equations [39]. We refer to [1, 30] for a rigorous derivation of the models and to [31] for a more recent review. Moreover, the Green-Naghdi system is one of the most widely applied models to perform numerical simulations of coastal flows [9,32]. In the equations, the flow is assumed to be irrotational or almost irrotational. This additional consideration yields significant simplifications, since the relevant information to describe the fluid motion is then concentrated on the interface. Zakharov [46] noticed that the irrotational equations enjoy a Hamiltonian structure and later, by means of the Dirichlet-Neumann operator, Craig and Sulem rewrote the Hamiltonian system as a close set of two dimensional evolution equations, cf. [10]. However, the Zakharov-Craig-Sulem approach is merely restricted to the irrotational setting which breaks down in the presence of rip currents or when underlying currents are present. A Hamiltonian formulation of the water waves equation in the presence of vorticity was introduced in [6] where the three dimensional vorticity is coupled with the evolution equation of the two-dimensional quantities of the classical Zakharov-Craig-Sulem formulation. Hence, it is not a priori obvious whether one can reduce the system to a two-dimensional closed set of equations.

Different approaches to deal with this difficulty have been developed in the literature. In particular, a novel strategy is proposed in [7], where the additional terms appearing in the momentum equation can be treated without appealing to the resolution of the three-dimensional vorticity equation. The strategy followed in [7], inspired by turbulence theory, hinges on deriving a cascade of equations which is actually finite at the precision of the model $\left(\mathcal{O}\left(\mu^{2}\right)\right.$-order) without any artificial closure. The resulting equations are an extension of the classical Green-Naghdi equations, where the non-hydrostatic pressure terms are affected by the interactions between the horizontal and vertical components of the vorticity. In conclusion, hydrodynamic shallow water models have been well studied and extensively used to describe fluid motions in 
oceans, with direct applications in coastal engineering [26,27]. Surprisingly enough, most of the electrically conducting fluids appearing in astrophysical plasmas, such as accretion disks, planetary atmospheres or stars, are in some sense thin. However, the role of magnetic fields when the fluid is electrically conducting is far from being well-understood.

The introduction of magnetic effects into the shallow water system was first proposed by Gilman [17] as a model for the solar tachocline. The tachocline, first coined by Spiegel and Zahn [40], is a very thin layer in the Sun of thickness of about five per-cent of the solar radius which bridges the transition region between the convective zone and the radiative zone [23]. The approximation of the shallow MHD equations presented by Gilman neglects $\mathcal{O}(\mu)$ order terms and hence can be understood as the magnetic analogue of the non-linear shallow water equations. Since their derivation, they have been investigated from a theoretical and modeling point of view. In particular, the Green-Naghdi equation including dispersive effects was studied in [14]. Studies on linear and non-linear waves have been treated in [38] and applications of the shear-flow instabilities in [28]. The shallow MHD system has also been shown to be hyperbolic and posseses a Hamiltonian structure $[11,13,36]$. Extensions from one-layer to multi-layers models of the shallow MHD equations experimenting with different stratification settings, were also documented in [24,47].

The main purpose of this article is to present asymptotic models for the shallow MHD equation (SMHD) with vorticity and magnetic current under a weak magnetic pressure assumption up to a precision of $\mathcal{O}\left(\mu^{3 / 2}\right)$. The derived models take into account the dispersive effects included in the work of [14] due to the non-hydrostatic pressure, but most importantly, also include shearing effects (which are assumed to be weakly sheared plasmas). Our strategy is influenced by the work in [7] for the water waves equations based on ideas reminiscent of turbulence theory. The main point relies on getting rid of the three-dimensional vorticity-current system, and instead look for two equations involving two dimensional key quantities to close the cascade equation: the shear velocity and the magnetic shear. Roughly speaking, those quantities represent the contributions of the horizontal vorticity and magnetic current in the horizontal momentum equation.

\subsection{Plan of the Paper}

In Sect. 2 we present the basic equations of the free-surface MHD problem. Section 3 is devoted to the asymptotic analysis of the free-surface MHD equation, where we first recast the formulation of the MHD problem by using the elevation-discharge variables. In particular, Sect. 3.2 deals with the non-dimensional averaged-formulation. An asymptotic description of the velocity field and magnetic field which depends on the velocity and magnetic shear term is performed in Sect. 3.2. Section 4 presents the different shallow asymptotic models in 1D and 2D. In particular, in Sect. 4.1, we first present the computation of the non-hydrostatic pressure and the different tensors. Later we derive an evolution equation for the velocity and magnetic shear Sect. 4.1.1 and provide a closure equation for the tensors in Sect. 4.1.2. In Sect. 4.2 we show the full 2D magnetic Green-Naghdi equations with shearing and compute its 
dispersive properties in Sect. 4.2.1. In Sect. 4.3, we handle the 1D magnetic GreenNaghdi equations with shearing, where several terms trivialize. Finally, a conclusion and future work perspectives are given in Sect. 5.

\subsection{Notation}

We will use the following notation throughout the manuscript:

- We denote by $d=1,2$ the horizontal dimension, by $X \in \mathbb{R}^{d}$ the horizontal coordinate and by $z$ the vertical variable.

- We use $\nabla$ to denote the gradient with respect to the horizontal variables and $\nabla_{X, z}$ is the full three-dimensional gradient operator. For any vector field $F \in \mathbb{R}^{3}$, we choose the convention $\left(\nabla_{X, z} F\right)_{i j}=\partial_{i} F_{j}$, for $i, j=1,2,3$. Moreover, the rotational and divergence operators are given by

$$
\operatorname{curl} F=\nabla_{X, z} \times F, \quad \operatorname{div} F=\nabla_{X, z} \cdot F .
$$

- For every vector field $F \in \mathbb{R}^{3}$ we denote by $F_{h}$ its horizontal component and by $F_{v}$ its vertical component. If the vector field $F \in \mathbb{R}^{2}$ we denote by $F^{\perp}=$ $\left(F_{1}, F_{2}\right)^{\perp}=\left(-F_{2}, F_{1}\right)$. Let $A \in \mathbb{R}^{2} \times \mathbb{R}^{2}$ be a matrix, then $A^{t}$ is the transpose of $A$. Let $A, B \in \mathbb{R}^{2} \times \mathbb{R}^{2}$ two matrices, we denote by $(A \cdot B)_{i j}=A_{i k} B_{k j}$ the standard matrices product. The divergence of a matrix $A \in \mathbb{R}^{2} \times \mathbb{R}^{2}$ is the vector $(\nabla \cdot A)_{i}=\sum_{j=1}^{2} \partial_{j} A^{j i}$ for $i=1,2$. For any two different vector fields $F, G \in \mathbb{R}^{2}$, we define the tensor product $(F \otimes G)_{i j}=F_{i} G_{j}$, as the usual outer product.

\section{The Basic Equations}

In this section we are concerned with a free-boundary problem of ideal incompressible magnetohydrodynamics. The problem consists in finding a variable domain $\Omega_{t}^{-}$ occupied by an electrically conducting homogeneous plasma, together with a velocity field $U=U(X, z, t)$, the scalar pressure $P=P(X, z, t)$ and the magnetic field $B=B(X, z, t)$ satisfying the equations of MHD. The elevation of the free surface is parametrized by the graph of a function $\xi(\cdot, t)$, and the non-moving bottom topography is parametrized by a time-independent function $-H_{0}+b(X)$ where $H_{0}$ represents the the depth of the plasma and $b$ the possible variation of the bottom, see Fig. 1. Therefore, the domain occupied by the plasma at time $t$ is

$$
\Omega_{t}^{-}=\left\{(X, z) \in \mathbb{R}^{d+1}:-H_{0}+b(X)<z<\xi(X, t)\right\} .
$$

The dynamics of the plasma region is governed by the following ideal incompressible MHD equations: 


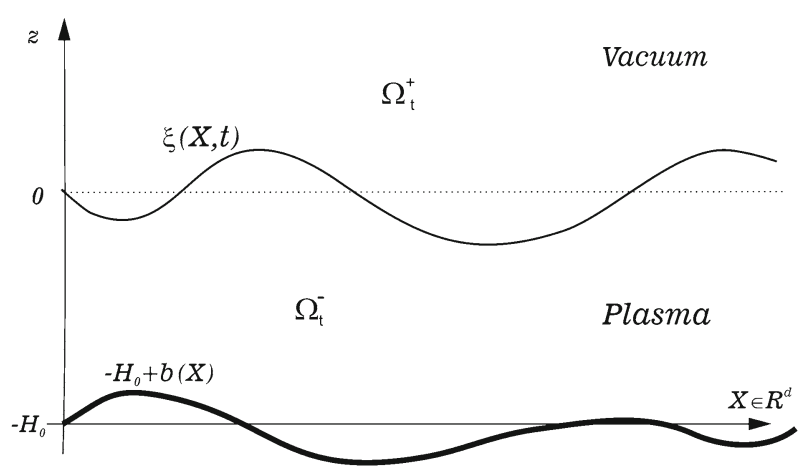

Fig. 1 The plasma-vacuum interface problem setting

$$
\begin{cases}\partial_{t} U+\left(U \cdot \nabla_{X, z}\right) U=-\frac{1}{\rho} \nabla_{X, z} P-g e_{z}+\frac{1}{\rho \mu_{0}}\left(\left(B \cdot \nabla_{X, z}\right) B-\frac{1}{2} \nabla_{X, z}|B|^{2}\right) & \text { in } \Omega_{t}^{-}, \\ \partial_{t} B+\left(U \cdot \nabla_{X, z}\right) B=\left(B \cdot \nabla_{X, z}\right) U & \text { in } \Omega_{t}^{-}, \\ \operatorname{div} B=0, \quad \operatorname{div} U=0 & \text { in } \Omega_{t}^{-},\end{cases}
$$

where the external forces due to gravity $g$ with $e_{z}=(0,0,1)$ are also taken into account. Above $\rho$ is the density assumed constant, and $\mu_{0}$ the magnetic permeability constant. The plasma is surrounded by a vacuum region,

$$
\Omega_{t}^{+}=\left\{(X, z) \in \mathbb{R}^{d+1}: z>\xi(X, t)\right\}
$$

at time $t$. Since, vacuum has no density, velocity or electric current, the pre-Maxwell dynamics apply $[4,19]$. In such case, the magnetic field $\widehat{B}$ is determined by the div-curl as follows:

$$
\begin{cases}\nabla_{X, z} \times \widehat{B}=0, & \text { in } \Omega_{t}^{+}, \\ \operatorname{div} \widehat{B}=0, & \text { in } \Omega_{t}^{+}\end{cases}
$$

The physical quantities of the plasma and vacuum region must satisfy non-trivial jump conditions connecting the fields across the interface (cf. [19] for a thorough exposition). The first boundary condition, the so-called kinematic boundary condition, is related to the fact that the plasma particles on the surface stay on the surface moving with normal velocity $V_{n}=U \cdot N$ given by

$$
\partial_{t} \xi-U_{\left.\right|_{\mathrm{s}}} \cdot N=0
$$

where $U(X, t)_{\left.\right|_{\mathrm{s}}}=U(X, t, \xi(X, t))$ and $N=(-\nabla \xi, 1)^{t}$. The second and third conditions, satisfied at the surface, are the pressure balance condition and magnetic jump continuity: 


$$
\begin{aligned}
{\left[\left[P+\frac{1}{2 \mu_{0}}|B|^{2}\right]\right] } & =0 \text { on }\{z=\xi(X, t)\}, \\
{\left[\left[B_{\left.\right|_{\mathrm{s}}} \cdot N\right]\right] } & =0
\end{aligned}
$$

where $[[f]]=\widehat{f}-f$ denotes the jump in a quantity across the surface. Hence,

$$
\begin{aligned}
P+\frac{1}{2 \mu_{0}}|B|^{2} & =\frac{1}{2 \mu_{0}}|\widehat{B}|^{2}, \quad \text { on } \quad\{z=\xi(X, t)\}, \\
B_{\left.\right|_{\mathrm{s}}} \cdot N & =\widehat{B}_{\left.\right|_{\mathrm{s}}} \cdot N .
\end{aligned}
$$

Finally, we also impose two boundary conditions at the bottom topography, assumed to be a perfect conducting and impermeable material, as follows:

$$
\begin{aligned}
& U_{l_{\mathrm{b}}} \cdot N_{b}=0, \\
& B_{\left.\right|_{\mathrm{b}}} \cdot N_{b}=0,
\end{aligned}
$$

where $U_{\left.\right|_{\mathrm{b}}}(X, t)=U\left(X, t,-H_{0}+b(X)\right), B_{\left.\right|_{\mathrm{b}}}(X, t)=B\left(X, t,-H_{0}+b(X)\right)$ and $N_{b}=(-\nabla b, 1)^{t}$.

In order to simplify the dynamics of the equations we will assume that the vacuum magnetic field $\widehat{B}$ is identically zero, which of course is a trivial solution of (2.4). In this particular case, the free boundary MHD problem is given by

$$
\begin{cases}\partial_{t} U+\left(U \cdot \nabla_{X, z}\right) U=-\frac{1}{\rho} \nabla_{X, z} P-g e_{z}+\frac{1}{\rho \mu_{0}}\left(\left(B \cdot \nabla_{X, z}\right) B-\frac{1}{2} \nabla_{X, z}|B|^{2}\right) & \text { in } \Omega_{t}^{-}, \\ \partial_{t} B+\left(U \cdot \nabla_{X, z}\right) B=\left(B \cdot \nabla_{X, z}\right) U & \text { in } \Omega_{t}^{-}, \\ \operatorname{div} B=0, \quad \operatorname{div} U=0 & \text { in } \Omega_{t}^{-},\end{cases}
$$

with boundary conditions

$$
\begin{aligned}
\partial_{t} \xi+U_{\left.\right|_{\mathrm{s}}} \cdot N & =0, \\
\left(P+\frac{1}{2 \mu_{0}}|B|^{2}\right)_{\left.\right|_{\mathrm{s}}} & =0, \\
B_{\left.\right|_{\mathrm{s}}} \cdot N & =0, \\
U_{\mathrm{l}_{\mathrm{b}}} \cdot N_{b} & =0, \\
B_{\left.\right|_{\mathrm{b}}} \cdot N_{b} & =0 .
\end{aligned}
$$

Remark 1 In (2.10b) we are neglecting the effects of the surface tension, which can be also incorporated into the model, cf. [8]. Moreover, we also neglect the Coriolis effects induced by planetary rotation. 


\section{The Averaged MHD Equations and Asymptotic Analysis}

In this section we will recast the MHD equations (2.9) using the so-called elevationdischarge formulation (cf. [26]) that proves to be very convenient in order to obtain and understand different asymptotic models. The central idea of the formulation is to get rid of the vertical variable by integrating vertically the horizontal component of the free surface MHD equation. To that purpose, let us denote by $U=U(X, z, t)=$ $\left(U_{h}(X, z, t), U_{v}(X, z, t)\right), B=B(X, z, t)=\left(B_{h}(X, z, t), B_{v}(X, z, t)\right)$ the horizontal and vertical component of the plasma velocity and magnetic field, respectively. Moreover, we introduce the horizontal velocity discharge $Q$,

$$
Q(X, t)=\int_{-H_{0}+b(X)}^{\xi(X, t)} U_{h}(X, z, t) \mathrm{d} z
$$

and the horizontal magnetic discharge $Q_{B}$,

$$
Q_{B}(X, t)=\int_{-H_{0}+b(X)}^{\xi(X, t)} B_{h}(X, z, t) \mathrm{d} z .
$$

Integrating vertically the horizontal component of equations (2.9) and using the boundary conditions $(2.10 \mathrm{a}),(2.10 \mathrm{c}),(2.10 \mathrm{~d})$ and $(2.10 \mathrm{e})$ gives

$$
\left\{\begin{array}{l}
\partial_{t} \xi+\nabla \cdot Q=0, \\
\partial_{t} Q+\nabla \cdot\left(\int_{-H_{0}+b}^{\xi} U_{h} \otimes U_{h}\right)+\frac{1}{\rho} \int_{-H_{0}+b}^{\xi} \nabla P_{B}=\frac{1}{\rho \mu_{0}} \nabla \cdot\left(\int_{-H_{0}+b}^{\xi} B_{h} \otimes B_{h}\right), \\
\partial_{t} Q_{B}+\nabla \cdot\left(\int_{-H_{0}+b}^{\xi} B_{h} \otimes U_{h}\right)=\nabla \cdot\left(\int_{-H_{0}+b}^{\xi} U_{h} \otimes B_{h}\right), \\
\nabla \cdot Q_{B}=0,
\end{array}\right.
$$

where $P_{B}=P+\frac{1}{2 \mu_{0}}|B|^{2}$ is the magnetic pressure. Next, we can decompose the magnetic pressure field into a hydrostatic magnetic pressure term $P_{B}^{h}$ and non-hydrostatic magnetic pressure term $P_{B}^{n h}$. It is easy to check that $\xi=0, U=0, B=0$ is a particular steady-state solution of equations (2.9) and, therefore, the vertical component of the first equation in (2.9) gives the following ordinary differential equation for $P_{B}$ :

$$
-\frac{1}{\rho} \partial_{z} P_{B}-g=0
$$

with boundary condition $P_{B_{\mid \mathrm{s}}}=0$. A direct computation shows that the solution is given by the hydrostatic magnetic pressure $P_{B}^{h}=-\rho g z$. Similarly when the fluid is not at rest, integrating the vertical component between $z$ and $\xi$ we have that $P_{B}=$ $\rho g(\xi-z)+P_{B}^{n h}$, where the non-hydrostatic magnetic pressure is given by

$$
P_{B}^{n h}(X, z, t)=\rho \int_{z}^{\xi(X, t)} \partial_{t} U_{v}+\left(U \cdot \nabla_{X, z}\right) U_{v}-\left(B \cdot \nabla_{X, z}\right) B_{v} \mathrm{~d} z .
$$


Plugging (3.5), we have that the evolution equations are given by

$$
\left\{\begin{array}{l}
\partial_{t} \xi+\nabla \cdot Q=0, \\
\partial_{t} Q+\nabla \cdot\left(\int_{-H_{0}+b}^{\xi} U_{h} \otimes U_{h}\right)+g h \nabla \xi \\
\quad+\frac{1}{\rho} \int_{-H_{0}+b}^{\xi} \nabla P_{B}^{n h}=\frac{1}{\rho \mu_{0}} \nabla \cdot\left(\int_{-H_{0}+b}^{\xi} B_{h} \otimes B_{h}\right), \\
\partial_{t} Q_{B}+\nabla \cdot\left(\int_{-H_{0}+b}^{\xi} B_{h} \otimes U_{h}\right)=\nabla \cdot\left(\int_{-H_{0}+b}^{\xi} U_{h} \otimes B_{h}\right), \\
\nabla \cdot Q_{B}=0,
\end{array}\right.
$$

where $h(X, t)$ is the plasma height, $h(X, t)=\xi(X, t)-H_{0}+b(X)$. In addition, following the original ideas introduced Teshukov in [41], we decompose the horizontal velocity and magnetic vector field as

$$
\begin{aligned}
& U_{h}(X, t, z)=\overline{U_{h}}(X, t)+U_{h}^{\star}(X, t, z), \\
& B_{h}(X, t, z)=\overline{B_{h}}(X, t)+B_{h}^{\star}(X, t, z),
\end{aligned}
$$

where for a general function $f(\cdot, t)$

$$
\bar{f}(X, t)=\frac{1}{h} \int_{-H_{0}+b(X)}^{\xi(X, t)} f(X, t, z) \mathrm{d} z
$$

with

$$
h(X, t)=\xi(X, t)+H_{0}-b(X) \quad \text { and } \quad f^{\star}(X, t, z)=f(X, t, z)-\bar{f}(X, t) .
$$

Using (3.7a)-(3.7b) into the equations, we have that

$$
\left\{\begin{array}{l}
\partial_{t} \xi+\nabla \cdot Q=0 \\
\partial_{t} Q+\nabla \cdot\left(\frac{1}{h} Q \otimes Q\right)+\nabla \cdot \mathcal{R}+g h \nabla \xi \\
\quad+\frac{1}{\rho} \int_{-H_{0}+b(X)}^{\xi(X, t)} \nabla P_{B}^{n h} \mathrm{~d} z=\frac{1}{\rho \mu_{0}} \nabla \cdot\left(\frac{1}{h} Q_{B} \otimes Q_{B}\right)+\frac{1}{\rho \mu_{0}} \nabla \cdot \mathcal{R}_{b} \\
\partial_{t} Q_{B}+\nabla \cdot\left(\frac{1}{h} Q_{B} \otimes Q\right)+\nabla \cdot \mathcal{R}_{m}=\nabla \cdot\left(\frac{1}{h} Q \otimes Q_{B}\right)+\nabla \cdot \mathcal{R}_{m}^{t} \\
\nabla \cdot Q_{B}=0
\end{array}\right.
$$

where

$$
\mathcal{R}:=\int_{-H_{0}+b(X)}^{\xi(X, t)} U_{h}^{\star} \otimes U_{h}^{\star} \mathrm{d} z \quad \text { and } \quad \mathcal{R}_{b}:=\int_{-H_{0}+b(X)}^{\xi(X, t)} B_{h}^{\star} \otimes B_{h}^{\star} \mathrm{d} z
$$




$$
\mathcal{R}_{m}:=\int_{-H_{0}+b(X)}^{\xi(X, t)} B_{h}^{\star} \otimes U_{h}^{\star} \mathrm{d} z \quad \text { and } \quad \mathcal{R}_{m}^{t}:=\int_{-H_{0}+b(X)}^{\xi(X, t)} U_{h}^{\star} \otimes B_{h}^{\star} \mathrm{d} z .
$$

We will refer to equations (3.10) as the averaged MHD equations. Although the equations are exact, they are not closed. Indeed, quick inspection of equations (3.10) reveal that the terms $\mathcal{R}, \mathcal{R}_{b}, \mathcal{R}_{m}$ and the non-hydrostatic magnetic pressure term (3.5) are non explicit in terms of the functions $\xi, Q$ and $Q_{B}$. Actually, as we will see in Sect. 4.1, they will be related with the vorticity $\omega=\nabla_{X, z} \times U$ and magnetic current $j=\frac{1}{\mu_{0}} \nabla_{X, z} \times B$ equations given by

$$
\left\{\begin{array}{l}
\partial_{t} \omega+\left(U \cdot \nabla_{X, z}\right) \omega-\frac{1}{\rho}\left(B \cdot \nabla_{X, z}\right) j=\left(\omega \cdot \nabla_{X, z}\right) U-\frac{1}{\rho}\left(j \cdot \nabla_{X, z}\right) B, \\
\partial_{t} j+\frac{1}{\mu_{0}}\left(U \cdot \nabla_{X, z}\right) j-\left(B \cdot \nabla_{X, z}\right) \omega=\frac{1}{\mu_{0}}\left(j \cdot \nabla_{X, z}\right) U-\left(\omega \cdot \nabla_{X, z}\right) B \\
-2 \sum_{i=1}^{3} \nabla_{X, z} U_{i} \times \nabla_{X, z} B_{i} .
\end{array}\right.
$$

\subsection{Non-Dimensional Free Boundary MHD Equations}

Studying the behaviour of the solutions of the full system is generally too complicated since it contains all the information of the dynamics. We will, therefore, simplify the terms which seem less important to us. To determine which terms are more relevant, we will use very well-known method based on the principle of dimensioning. We non-dimensionalize the equations by using several characteristic lengths of the problem, namely the following: the typical amplitude of the waves $a_{s}$, the typical depth $H_{0}$, the typical horizontal scale $L$ and the order of bottom variations $a_{b}$. With these characteristic scales we can construct three different dimensionless parameters:

$$
\mu=\frac{H_{0}^{2}}{L^{2}}, \epsilon=\frac{a}{H_{0}}, \beta=\frac{a_{b}}{H_{0}},
$$

where $\epsilon$ is called the amplitude parameter, $\beta$ the topography parameter and $\mu$ the shallowness parameter.

Remark 2 For simplicity we have assumed here that the horizontal scale $L$ is the same in both the longitudinal $L_{x}$ and transversal $L_{y}$ direction; however, this could also be considered into the modelling yielding a new parameter $\gamma=\frac{L_{x}}{L_{y}}$ called transversality parameter [26]. Since the main goal of this article is to derive shallow models we will assume that $\mu \ll 1$, but not other smallness assumption will be done. In particular, to lighten the equations we will assume that $\epsilon=1$, this is without loss of generality we assume that $H_{0}=a$.

Furthermore, another important parameter which measures the ratio of the pressure $P$ to the magnetic pressure $\frac{|B|^{2}}{2 \mu_{0}}$ is the so-called plasma- $\beta$ coefficient. ${ }^{1}$ Magnetic field

\footnotetext{
${ }^{1}$ Notice that the $\beta$-plasma parameter should not be confused with the topography parameter, although we have denoted them with the same letter. Since this is perfectly clear from the context we prefer not to include new terminology.
} 
strength in the solar tachocline in the range of $10^{4}-10^{5} \mathrm{G}$ (Gaussian units) corresponds to magnetic pressures up to $4 \times 10^{7} \mathrm{~Pa}$ which gives plasma- $\beta$ of the order above $10^{5}$ (cf. [49]). Hence, it is licit to regard the influence of the magnetic field on the equilibrium as a small perturbation. Taking this into account, we will then assume that the plasma$\beta$ is large and in particular of order $\mathcal{O}\left(\frac{1}{\mu}\right)$, i.e. $\beta=\frac{P}{\frac{B^{2}}{2 \mu_{0}}}=\mathcal{O}\left(\frac{1}{\mu}\right)$. In another words, we will derive models in the case of a weak magnetic field.

With the before-mentioned parameters and considerations, we will define the following dimensionless variables:

$$
\widetilde{z}=\frac{z}{H_{0}}, \quad \tilde{X}=\frac{X}{L}, \quad \tilde{t}=\frac{t}{\frac{L}{\sqrt{g H_{0}}}},
$$

and dimensionless unknown functions

$$
\left\{\begin{array}{l}
\widetilde{\xi}=\frac{\xi}{a}, \quad \widetilde{b}=\frac{b}{a_{b}} \quad \widetilde{U}_{h}=\frac{U_{h}}{a \sqrt{\frac{g}{H_{0}}}}, \quad \widetilde{U_{v}}=\frac{U_{v}}{\frac{a H_{0}}{L} \sqrt{\frac{g}{H_{0}}}} \\
\widetilde{B_{h}}=\frac{B_{h}}{a \sqrt{\frac{\mu \mu_{0} \rho g}{H_{0}}}}, \quad \widetilde{B_{v}}=\frac{B_{v}}{\frac{a H_{0}}{L} \sqrt{\frac{\mu_{0} \rho}{H_{0}}}}, \quad \widetilde{P}=\frac{P}{\rho g H_{0}} .
\end{array}\right.
$$

With this new variables at hand, we denote by

$$
\left\{\begin{array}{l}
\widetilde{U}=\left(\widetilde{U_{h}}, \widetilde{U_{v}}\right)^{t}, \quad \widetilde{B}=\left(\sqrt{\mu} \widetilde{B_{h}}, \widetilde{B_{v}}\right)^{t}, \widetilde{P_{B}}=\widetilde{P}+\frac{\mu}{2}\left({\widetilde{B_{h}}}^{2}+{\widetilde{B_{v}}}^{2}\right) \\
\widetilde{\nabla}=\left(\nabla_{\widetilde{X}}, \partial_{\bar{z}}\right)^{t} \quad \text { and } \widetilde{\operatorname{div}}=\widetilde{\nabla} \cdot
\end{array}\right.
$$

the free boundary MHD equations (2.9) are given in dimensionless form by

$$
\left\{\begin{array}{l}
\partial_{\tilde{t}} \widetilde{U}+\widetilde{U} \cdot \widetilde{\nabla} \widetilde{U}=-\left(\widetilde{\nabla} \widetilde{P_{B}}+e_{z}\right) \cdot\left(1, \frac{1}{\mu}\right)+\widetilde{B} \cdot \widetilde{\nabla} \widetilde{B}, \\
\partial_{\tilde{t}} \widetilde{B}+\widetilde{U} \cdot \widetilde{\nabla} \widetilde{B}=\widetilde{B} \cdot \widetilde{\nabla} \widetilde{U}, \\
\widetilde{\operatorname{div}} \widetilde{U}=0, \quad \widetilde{\operatorname{div}} \widetilde{B}=0,
\end{array}\right.
$$

posed in the dimensionless fluid domain,

$$
\Omega(\widetilde{t})^{-}=\left\{(\tilde{X}, \widetilde{z}) \in \mathbb{R}^{d+1}:-1+\beta \widetilde{b}(\tilde{X})<\widetilde{z}<\widetilde{\xi}(\widetilde{X}, \widetilde{t})\right\}
$$


In the same way, the boundary conditions in dimensionless form are given by

$$
\left\{\begin{array}{l}
\partial_{\tilde{t} \tilde{\xi}}-\widetilde{U}_{\left.\right|_{\tilde{z}=\tilde{\xi}}} \cdot N=0, \\
\widetilde{B}_{\left.\right|_{\tilde{z}=\tilde{\xi}}} \cdot N=0, \\
\left.\widetilde{P}_{B}\right|_{\tilde{z}=\tilde{\xi}}=0, \\
\widetilde{U}_{\left.\right|_{\tilde{z}=-1+\beta \tilde{b}}} \cdot N_{b}=0, \\
\widetilde{B}_{\left.\right|_{\tilde{z}=-1+\beta \tilde{b}}} \cdot N_{b}=0,
\end{array}\right.
$$

with $N=\left(\begin{array}{c}-\widetilde{\nabla} \widetilde{\xi} \\ 1\end{array}\right), N_{b}=\left(\begin{array}{c}-\beta \widetilde{\nabla} \widetilde{b} \\ 1\end{array}\right)$. It must be emphasized that all the tilde variables (besides the vertical magnetic field $\widetilde{B_{v}}$ which is $\mathcal{O}(\sqrt{\mu})$ ) are $\mathcal{O}(1)$ with respect to the shallow parameter $\mu$. In a similar way, the dimensionless version of the averaged MHD equations (3.10) are given by

$$
\left\{\begin{array}{l}
\partial_{\tilde{t}} \tilde{\xi}+\widetilde{\nabla} \cdot \widetilde{Q}=0, \\
\partial_{\tilde{t}} \widetilde{Q}+\widetilde{\nabla} \cdot\left(\frac{1}{\widetilde{h}} \widetilde{Q} \otimes \widetilde{Q}\right)+\widetilde{\nabla} \cdot \widetilde{\mathcal{R}}+\widetilde{h} \widetilde{\nabla} \widetilde{\xi} \\
\quad+\int_{-1+\beta \widetilde{\xi}}^{\widetilde{\nabla} P_{B}^{n h}} d \widetilde{z}=\mu \widetilde{\nabla} \cdot\left(\frac{1}{\widetilde{h}} \widetilde{Q_{B}} \otimes \widetilde{Q_{B}}\right)+\mu \widetilde{\nabla} \cdot \widetilde{\mathcal{R}_{b}}, \\
\partial_{\tilde{t}} \widetilde{Q_{B}}+\widetilde{\nabla} \cdot\left(\frac{1}{\widetilde{h}} \widetilde{Q_{B}} \otimes \widetilde{Q}\right)+\widetilde{\nabla} \cdot \widetilde{\mathcal{R}_{m}}=\widetilde{\nabla} \cdot\left(\frac{1}{\widetilde{h}} \widetilde{Q} \otimes \widetilde{Q_{B}}\right)+\widetilde{\nabla} \cdot \widetilde{\mathcal{R}_{m}^{t}}, \\
\widetilde{\nabla} \cdot \widetilde{Q_{B}}=0,
\end{array}\right.
$$

where $\widetilde{Q}=\frac{Q}{H_{0} \sqrt{g H_{0}}}, \widetilde{Q_{B}}=\frac{Q_{B}}{H_{0} \sqrt{\mu \mu_{0} g \rho H_{0}}}$ are the dimensionless velocity and magnetic discharge, $\widetilde{h}=-1+\beta \widetilde{b}+\widetilde{\xi}$ the dimensionless plasma height and the dimensionless tensors and non-hydrostatic terms are given by the following:

$$
\begin{aligned}
\widetilde{P_{B}^{n h}} & =\mu \int_{\widetilde{z}}^{\tilde{\xi}}\left(\partial_{\tilde{t}} \widetilde{U_{v}}+\widetilde{U_{h}} \cdot \widetilde{\nabla} \widetilde{U}_{v}+\widetilde{U_{v}} \partial_{\bar{z}} \widetilde{U_{v}}-\sqrt{\mu} \widetilde{B_{h}} \cdot \widetilde{\nabla} \widetilde{B_{v}}+\widetilde{B_{v}} \partial_{\bar{z}} \widetilde{B_{v}}\right), \\
\widetilde{\mathcal{R}} & =\int_{-1+\beta \widetilde{b}}^{\widetilde{\xi}} \widetilde{U_{h}^{\star}} \otimes \widetilde{U_{h}^{\star}} d \widetilde{z} \text { and } \widetilde{\mathcal{R}_{b}}=\int_{-1+\beta \widetilde{\xi}}^{\widetilde{\xi}} \widetilde{B_{h}^{\star}} \otimes \widetilde{B_{h}^{\star}} d \widetilde{z}, \\
\widetilde{\mathcal{R}_{m}} & =\int_{-1+\beta \widetilde{b}}^{\tilde{\xi}} \widetilde{B_{h}^{\star}} \otimes \widetilde{U_{h}^{\star}} d \widetilde{z} .
\end{aligned}
$$

Analogously as in the case the free-surface Euler equation in the presence of nontrivial vorticity [6,7], we require a true comprehension of the behaviour of the vorticity $\omega$ and the current $j$. We will only treat the case of weakly sheared flows (using the terminology of [41] and later also used in $[6,7,34,35]$ ), that is we will rescale the vorticity and the current as follows: 


$$
\widetilde{\omega}=\frac{L}{a} \sqrt{\frac{H_{0}}{g}} \omega, \quad \tilde{j}=\frac{L}{a} \sqrt{\frac{H_{0}}{g \mu_{0} \rho}} j
$$

where the new variables $\widetilde{\omega}$ and $\tilde{j}$ are $\mathcal{O}(\sqrt{\mu})$, so we are in the sheared weak flow regime.

Remark 3 Deriving different models based on different assumptions on the strength of the vorticity and magnetic current is also possible. However, here we treat the case of weakly sheared plasmas, $\widetilde{\omega}$ and $\widetilde{j}$ are $\mathcal{O}(\sqrt{\mu})$. In [15,27] models with stronger rotational effects are studied for the water waves problem: however, whether this quantities remain of the same order during the time evolution of the flow is not clear. In [6] it is rigorously shown that for the Green-Naghdi equation with vorticity, weakly sheared flows $\widetilde{\omega}=\mathcal{O}(\sqrt{\mu})$ remain of that order during the evolution. We notice that in this article, we do not provide such a rigorous result, but only derive the asymptotic models.

Remark 4 From now on until the end of the paper, we will omit the tilde notation on the different variables and unknowns to lighten the derivation and enhance exposition clearness.

\subsection{Asymptotic Expansion of the Inner Velocity and Magnetic Field}

In this section, we will derive inner asymptotic description of the velocity field $U$ and magnetic field $B$. First, let us consider the the following boundary value problem satisfied by the velocity field:

$$
\begin{cases}\operatorname{curl} U=\omega & \text { in } \Omega \\ \operatorname{div} U=0 & \text { in } \Omega \\ U_{\left.\right|_{z=-1+\beta b}} \cdot N_{b}=0 & \text { at the bottom }\end{cases}
$$

The expansion coincides with the one in [7] for the water waves equation (where $B=0$ ) and is given by

$$
\begin{aligned}
U_{h} & :=\overline{U_{h}}+U_{h}^{\star}=\overline{U_{h}}+\sqrt{\mu} U_{s h}^{\star}+\mathcal{O}(\mu) \\
U_{v} & =\left(\nabla \cdot \overline{U_{h}}(1+z-\beta b)\right)+\mathcal{O}(\sqrt{\mu})
\end{aligned}
$$

The shear velocity $U_{s h}$ is given by

$$
U_{s h}=\int_{z}^{\xi} \omega_{h}^{\perp}, \quad \text { where } U_{s h}^{\star}=\left(\int_{z}^{\xi} \omega_{h}^{\perp}\right)^{\star}=\int_{z}^{\xi} \omega_{h}^{\perp}-\frac{1}{h} \int_{-1+\beta b}^{\xi} \int_{z}^{\xi} \omega_{h}^{\perp}
$$

This suggests that the fluctuations of the horizontal velocity $U_{h}^{\star}=U_{h}-\overline{U_{h}}$ is mainly due to the influence of the vorticity contributing at order $\mathcal{O}(\sqrt{\mu})$, whilst the contribution of the vorticity to the vertical component is much smaller appearing in $\mu$-higher orders that we neglect at the precision of this model. 
Next, we will consider the expansion of the magnetic field $B$, which satisfies the following:

$$
\begin{cases}\operatorname{curl} B=j & \text { in } \Omega, \\ \operatorname{div} B=0 & \text { in } \Omega, \\ B_{\left.\right|_{z=\xi}} \cdot N=0 & \text { at the surface, } \\ B_{\left.\right|_{z=-1+\beta b}} \cdot N_{b}=0 & \text { at the bottom. }\end{cases}
$$

Notice that the boundary value problem for the magnetic field $B$, is completely determined by the magnetic current $j$. In contrast with the velocity field where the irrotational part of the $U$ is determined from the tangential component at the surface, the magnetic field does not have this extra degree of freedom (cf. Remark 3 in [7]). Taking the horizontal and vertical component of equations we have that

$$
\left\{\begin{array}{l}
\partial_{z} B_{h}-\sqrt{\mu} \nabla B_{v}=-j_{h}^{\perp} \\
\sqrt{\mu} \nabla^{\perp} \cdot B_{h}=j_{v} \\
\sqrt{\mu} \nabla \cdot B_{h}+\partial_{z} B_{v}=0 \\
-\sqrt{\mu} \nabla \xi \cdot B_{h}+B_{v}=0, \\
-\beta \sqrt{\mu} \nabla b \cdot B_{h}+B_{v}=0
\end{array}\right.
$$

We plug the following Ansatz:

$$
\begin{aligned}
& B_{h}=B_{h}^{0}+\mathcal{O}(\sqrt{\mu}) \\
& B_{v}=B_{v}^{0}+\sqrt{\mu} B_{v}^{1}+\mathcal{O}(\mu)
\end{aligned}
$$

and write $j_{v}=j_{v}^{0}+\sqrt{\mu} j_{v}^{1}$ to be consistent with the fact that div $j=0$. We infer that $j_{v}^{0} \equiv 0$ and $B_{v}^{0}$ satisfies

$$
\begin{cases}\partial_{z} B_{v}^{0}=0 & \text { in } \Omega, \\ B_{v}^{0}=0 & \text { at the surface, } \\ B_{v}^{0}=0 & \text { at the bottom, }\end{cases}
$$

and hence a simple integration yields that $B_{v}^{0} \equiv 0$. The functions $\left(B_{h}^{0}, B_{v}^{1}\right)$ solve the following system:

$$
\left\{\begin{array}{l}
B_{h}^{0}(x, y, z)=B_{h}^{0}(x, y, \xi)+\int_{z}^{\xi} j_{h}^{\perp}, \\
B_{v}^{1}(x, y, z)=B_{v}^{1}(x, y,-1+\beta b)-\int_{-1+\beta b}^{z} \nabla \cdot B_{h}^{0} \mathrm{~d} z, \\
B_{v}^{1}(x, y, \xi)=B_{h}^{0}(x, y, \xi) \nabla \xi \\
B_{v}^{1}(x, y,-1+\beta b)=\beta B_{h}^{0}(x, y,-1+\beta b) \nabla b .
\end{array}\right.
$$

Plugging the first equation in (3.30) into the second equation, evaluating at $z=\xi$ and using the boundary conditions we have that 


$$
\left\{\begin{array}{l}
B_{h}^{0}(x, y, z)=B_{h}^{0}(x, y, \xi)+\int_{z}^{\xi} j_{h}^{\perp} \quad \text { in } \Omega \\
\nabla \cdot\left(h B_{h}^{0}(x, y, \xi)\right)=-\nabla \cdot \int_{-1+\beta b}^{\xi} \int_{z}^{\xi} j_{h}^{\perp}, \\
\nabla^{\perp} \cdot B_{h}^{0}(x, z)=j_{v}^{1} .
\end{array}\right.
$$

with $h=-1+\beta b+\xi$. Therefore, using the second equation in (3.31) gives

$$
B_{h}^{1}(x, y, \xi)=-\frac{1}{h} \int_{-1+\beta b}^{\xi} \int_{z}^{\xi} j_{h}^{\perp}+\frac{1}{h} \nabla^{\perp} \phi^{1}(x, y)
$$

for some potential function $\phi(x, y)$. Hence,

$$
B_{h}^{1}(x, y, z)=\int_{z}^{\xi} j_{h}^{\perp}-\frac{1}{h} \int_{-1+\beta b}^{\xi} \int_{z}^{\xi} j_{h}^{\perp}+\frac{1}{h} \nabla^{\perp} \phi(x, y)=B_{s h}^{\star}+\frac{1}{h} \nabla^{\perp} \phi(x, y),
$$

where the magnetic shear $B_{s h}^{\star}$ is defined as

$$
B_{s h}^{\star}(x, y, z)=\int_{z}^{\xi} j_{h}^{\perp}-\frac{1}{h} \int_{-1+\beta b}^{\xi} \int_{z}^{\xi} j_{h}^{\perp} .
$$

The unknown potential function $\phi(x, y)$ satisfies also that

$$
\nabla^{\perp} \cdot\left(\frac{1}{h} \nabla^{\perp} \phi\right)=j_{v}^{1}(x, y, z)-\nabla^{\perp} \cdot B_{s h}^{\star} .
$$

One could also calculate the explicit expression of $B_{v}^{1}(x, y, z)$ using the second equation in (3.30) and (3.32). However, at the order of precision of the derived models this will be neglected since it will give rise to terms of order $\mathcal{O}\left(\mu^{3 / 2}\right)$.

Therefore, we have that the magnetic field $B$ can be describe by the following asymptotic expansion:

$$
\begin{aligned}
B_{h} & =\overline{B_{h}}+B_{s h}^{\star}+\mathcal{O}(\sqrt{\mu}) \\
B_{v} & =\mathcal{O}(\sqrt{\mu}),
\end{aligned}
$$

where we have denoted $\overline{B_{h}}=\frac{1}{h} \nabla^{\perp} \phi(x, y)$ and $\overline{B_{h}}$ satisfies

$$
\nabla^{\perp} \cdot\left(\overline{B_{h}}\right)=j_{v}^{1}(x, y, z)-\nabla^{\perp} \cdot B_{s h}^{\star} .
$$




\section{The Magnetic Green-Naghdi Equation with Shearing}

We deal here with the derivation of the magnetic Green-Naghdi with shearing type ${ }^{2}$ of equation in the two-dimensional case $(d=2)$; however, we will only work with models up to precision $\mathcal{O}\left(\mu^{3 / 2}\right)$. Structural complications arise in order to close the two-dimensional cascade of equations if we want to push the expansion further to full order $\mathcal{O}\left(\mu^{2}\right)$. Those difficulties are due to the strong coupling between the magnetic field and velocity field.

Before presenting the derivation of the magnetic Green-Naghdi equation with shearing let us recall the so-called nonlinear shallow MHD equations introduced by Gilman [17]. Without the magnetic pressure assumption, the nonlinear shallow MHD equations are an approximation of order $\mathcal{O}(\mu)$ of the full MHD equations (3.19), in the sense that all the terms of order $\mathcal{O}(\mu)$ are dropped. In particular, the non-hydrostatic pressure and turbulent effects coming from the tensors are not present in this first approximation. Therefore, neglecting terms of order $\mathcal{O}(\mu)$, Gilman obtained the following system of equations:

$$
\left\{\begin{array}{l}
\partial_{t} \xi+\nabla \cdot\left(h \overline{U_{h}}\right)=0, \\
\partial_{t}\left(h \overline{U_{h}}\right)+\nabla \cdot\left(h \overline{U_{h}} \otimes \overline{U_{h}}\right)=-h \nabla \xi+\nabla \cdot\left(h \overline{B_{h}} \otimes \overline{B_{h}}\right) \\
\partial_{t}\left(h \overline{B_{h}}\right)+\nabla \cdot\left(h \overline{U_{h}} \otimes \overline{B_{h}}\right)=\nabla \cdot\left(h \overline{B_{h}} \otimes \overline{U_{h}}\right)
\end{array}\right.
$$

for $t \geq 0, x \in \mathbb{R}^{2}, h=1+\xi-\beta b$. The system (4.1) can be understood as the magnetic MHD version of the well-known nonlinear shallow water equations [33]. Notice that under the weak pressure assumptions the term $\nabla \cdot\left(h \overline{B_{h}} \otimes \overline{B_{h}}\right)$ is $\mathcal{O}(\mu)$ and hence the equation reduces to the usual classical nonlinear shallow water equation. Later, in [14], the author derived the magnetic Green-Naghdi equation (without shearing, no bottom variations and weak pressure magnetic assumption) introducing the dispersive effects coming from the non-hydrostatic pressure given by

$$
\left\{\begin{array}{l}
\partial_{t} \xi+\nabla \cdot\left(h \overline{U_{h}}\right)=0, \\
\partial_{t}\left(h \overline{U_{h}}\right)+\nabla \cdot\left(h \overline{U_{h}} \otimes \overline{U_{h}}\right)+h \nabla \xi-\nabla \cdot\left(h \overline{B_{h}} \otimes \overline{B_{h}}\right)=\mu \mathcal{A} \\
\partial_{t}\left(h \overline{B_{h}}\right)+\nabla \cdot\left(h \overline{U_{h}} \otimes \overline{B_{h}}\right)=\nabla \cdot\left(h \overline{B_{h}} \otimes \overline{U_{h}}\right),
\end{array}\right.
$$

where

$$
\mathcal{A}=\frac{1}{3} \nabla\left(h^{2} \mathcal{D}\left(h \nabla \cdot \overline{U_{h}}\right)+h^{3}\left(\nabla \cdot \overline{B_{h}}\right)-h^{3} \overline{B_{h}} \cdot \nabla\left(\nabla \cdot \overline{B_{h}}\right)\right)
$$

and $\mathcal{D}=\left(\partial_{t}+\overline{U_{h}} \cdot \nabla\right)$ is the material operator. As the reader will notice hereafter, in the weak magnetic pressure regime, the magnetic dispersive terms in $\mathcal{A}$ are negligible (see Eq. (4.6)).

\footnotetext{
2 We have coined the equation magnetic Green-Naghdi equation with shearing due to the analogy with the Green-Naghdi equations with vorticity for water waves. However, it must be noticed that we only deal with precision up to $\mathcal{O}\left(\mu^{3 / 2}\right)$.
} 
Remark 5 The non-linear shallow MHD equations (4.1) were first derived by [17], and studied since their derivation intensively, see [24,29] for recent reviews. In [13], the author studied the hyperbolic character of the non-linear shallow MHD equations. General theory for symmetric hyperbolic systems (cf. [3]) assures the local wellposedness for initial data in Sobolev spaces $H^{s}\left(\mathbb{R}^{d}\right)$ with $s>1+\frac{d}{2}$. In recent paper, Trakhinin [44] investigated the structural stability of shock waves and current-vortex sheets in the non-linear shallow MHD equation (4.1).

\subsection{Computation of the Non-Hydrostatic and Tensors Contributions}

Next, we will compute the contributions of the non-hydrostatic terms (3.20) and the tensors (3.21)-(3.22) up to a precision of $\mathcal{O}\left(\mu^{3 / 2}\right)$. Using the asymptotic expansion (3.24) and (3.34) into (3.21) and (3.22) yields

$$
\begin{aligned}
\nabla \cdot \mathcal{R} & =\nabla \cdot \int_{-1+\beta b}^{\xi} U_{h}^{\star} \otimes U_{h}^{\star} \mathrm{d} z=\mu \nabla \cdot \int_{-1+\beta b}^{\xi} U_{s h}^{\star} \otimes U_{s h}^{\star}+\mathcal{O}\left(\mu^{3 / 2}\right), \\
\nabla \cdot \mathcal{R}_{b} & =\nabla \cdot \int_{-1+\beta b}^{\xi} B_{h}^{\star} \otimes B_{h}^{\star} \mathrm{d} z=\nabla \cdot \int_{-1+\beta b}^{\xi} B_{s h}^{\star} \otimes B_{s h}^{\star}+\mathcal{O}(\sqrt{\mu}), \\
\nabla \cdot \mathcal{R}_{m} & =\nabla \cdot \int_{-1+\beta b}^{\xi} B_{h}^{\star} \otimes U_{h}^{\star} \mathrm{d} z=\sqrt{\mu} \nabla \cdot \int_{-1+\beta b}^{\xi} B_{s h}^{\star} \otimes U_{s h}^{\star}
\end{aligned}
$$

Notice that the term $\nabla \cdot \mathcal{R}_{m}=\mathcal{O}(\sqrt{\mu})$ appears only in the evolution equation of $\widetilde{Q_{B}}$. Due to the weak magnetic assumption, all the terms $\mathcal{O}(\sqrt{\mu})$ in the evolution equation of $\widetilde{Q_{B}}$ can be neglected (since they are actually $\mathcal{O}\left(\mu^{3 / 2}\right)$ contributions). The same reasoning follows to check that we can neglect the terms of order $\mathcal{O}(\sqrt{\mu})$ appearing when deriving the evolution equation of $\mathcal{R}_{b}$ below (cf. Sect. 4.1.2).

Similarly, we can compute the non-hydrostatic magnetic pressure contributions. Dropping $\mathcal{O}\left(\mu^{3 / 2}\right)$ terms we get

$$
\begin{aligned}
& \int_{-1+\beta b}^{\xi} \nabla P_{B}^{n h} \\
& =\mu \int_{-1+\beta b}^{\xi} \nabla \int_{z}^{\xi}\left(\partial_{t} U_{v}+U_{h} \cdot \nabla U_{v}+U_{v} \partial_{z} U_{v}-\sqrt{\mu} B_{h} \cdot \nabla B_{v}+B_{v} \partial_{z} B_{v}\right) \\
& =-\mu \int_{-1+\beta b}^{\xi} \nabla \int_{z}^{\xi}\left(\partial_{t}\left(\nabla \cdot\left(\overline{U_{h}}(1+z-\beta b)\right)\right)+\overline{U_{h}} \cdot \nabla\left(\nabla \cdot\left(\overline{U_{h}}(1+z-\beta b)\right)\right)\right. \\
& \left.\quad+\left(\nabla \cdot\left(\overline{U_{h}}(1+z-\beta b)\right)\right) \partial_{z}\left(\nabla \cdot\left(\overline{U_{h}}(1+z-\beta b)\right)\right)\right)+\mathcal{O}\left(\mu^{3 / 2}\right) \\
& =\mu h \mathfrak{F}[h, b]\left(\partial_{t} \overline{U_{h}}+\overline{U_{h}} \nabla \cdot \overline{U_{h}}\right)+\mu h \mathfrak{D}[h, b]\left(\overline{U_{h}}\right)+\mathcal{O}\left(\mu^{3 / 2}\right),
\end{aligned}
$$

where the linear operator $\mathfrak{F}[h, b]$ and the quadratic form $\mathfrak{D}[h, b](\cdot)$ are defined for all smooth enough $\mathbb{R}^{2}$ vector valued functions $\mathrm{W}$ by 


$$
\begin{aligned}
\mathfrak{F}[h, b] W & =\mathfrak{R}_{1}[h, b](\nabla \cdot W)+\beta \mathfrak{R}_{2}[h, b](\nabla b \cdot W) \\
\mathfrak{D}[h, b](W) & =-2 \mathfrak{R}_{1}\left(\partial_{x} W \cdot \partial_{y} W^{\perp}+(\nabla \cdot W)^{2}\right)+\beta \mathfrak{R}_{2}(W \cdot(W \cdot \nabla) \nabla b),
\end{aligned}
$$

with, for all smooth enough scalar-valued functions $f$

$$
\mathfrak{R}_{1}[h, b] f=-\frac{1}{3 h} \nabla\left(h^{3} f\right)-\beta \frac{h}{2} f \nabla b, \quad \mathfrak{R}_{2}[h, b] f=\frac{1}{2 h} \nabla\left(h^{2} f\right)+\beta f \nabla b .
$$

For the sake of clarity, we often write $\mathfrak{F}, \mathfrak{D}, \mathfrak{R}_{1}, \mathfrak{R}_{1}$ instead of $\mathfrak{F}[h, b], \mathfrak{D}[h, b], \mathfrak{R}_{1}[h, b]$, $\mathfrak{R}_{1}[h, b]$. We have derived the non-hydrostatic magnetic pressure contributions using the operators $\mathfrak{F}$ and $\mathfrak{D}$ which we borrowed from the formulation in [5]. Therefore, inserting the expressions (4.3)-(4.5) and the magnetic pressure equation (4.6) into the equations (3.19), noticing that $\partial_{t} h+\nabla \cdot\left(h \overline{U_{h}}\right)=0$ and $\nabla \cdot\left(h \overline{B_{h}}\right)=0$, we infer that

$$
\left\{\begin{array}{l}
\partial_{t} \xi+\nabla \cdot\left(h \overline{U_{h}}\right)=0, \\
(1+\mu \mathfrak{F})\left(\partial_{t} \overline{U_{h}}+\overline{U_{h}} \cdot \nabla \overline{U_{h}}\right)+\nabla \xi+\mu \mathfrak{D}\left(\overline{U_{h}}\right)+\mu \frac{1}{h} \nabla \cdot \mathcal{R}=\mu \overline{B_{h}} \cdot \nabla \overline{B_{h}}+\mu \frac{1}{h} \nabla \cdot \mathcal{R}_{b} \\
\partial_{t} \overline{B_{h}}+\overline{U_{h}} \cdot \nabla \overline{B_{h}}=\overline{B_{h}} \cdot \nabla \overline{U_{h}},
\end{array}\right.
$$

where

$$
\mathcal{R}=\int_{-1+\beta b}^{\xi} U_{s h}^{\star} \otimes U_{s h}^{\star} \mathrm{d} z, \quad \mathcal{R}_{b}=\int_{-1+\beta b}^{\xi} B_{s h}^{\star} \otimes B_{s h}^{\star} \mathrm{d} z
$$

Hence, to close the equations we have find closure equations for the quantities $\mathcal{R}, \mathcal{R}_{b}$. To that purpose we have first to derive an evolution equation $U_{s h}^{\star}$ in (3.26) and magnetic shear $B_{s h}^{\star}$ defined in (3.33). With this equations at hand, we will be able to find the closure equations and close the system.

\subsubsection{Evolution Equation for $U_{s h}^{\star}$ and $B_{s h}^{\star}$}

To derive the evolution equation for $U_{s h}^{\star}$ and $B_{s h}^{\star}$, we make use of the evolution equations satisfied by the vorticity and current. Recall that the dimensionless vorticity and current in the case of weakly sheared flows is given by

$$
\widetilde{\omega}=\frac{L}{a} \sqrt{\frac{H_{0}}{g}} \omega, \quad \widetilde{j}=\frac{L}{a} \sqrt{\frac{H_{0}}{\mu_{0} g \rho}} j .
$$

Hence the dimensionless vorticity-current equations of (3.11), omitting the tilde notation, reads

$$
\left\{\begin{array}{l}
\partial_{t} \omega+(U \cdot \nabla) \omega-(B \cdot \nabla) j=(\omega \cdot \nabla) U-(j \cdot \nabla) B \\
\partial_{t} j+(U \cdot \nabla) j-(B \cdot \nabla) \omega=(j \cdot \nabla) U-(\omega \cdot \nabla) B-2 \sum_{i=1}^{3} \nabla U_{i} \times \nabla B_{i} .
\end{array}\right.
$$


Let us first derive an evolution equation for the shear velocity $U_{s h}^{\star}$. The computations are an adaptation of Sect. 2.3 in [7] .We recall the main steps, highlighting the new modifications. The horizontal component of the vorticity equation (4.8) is given by

$$
\begin{aligned}
& \partial_{t} \omega_{h}+U_{h} \cdot \nabla \omega_{h}+U_{v} \partial_{z} \omega_{h}-\sqrt{\mu} B_{h} \cdot \nabla j_{h}-B_{v} \partial_{z} j_{h} \\
& =\omega_{h} \cdot \nabla U_{h}+\frac{1}{\sqrt{\mu}} \omega_{v} \partial_{z} U_{h}-\sqrt{\mu}\left(j_{h} \cdot \nabla B_{h}-j_{v} \partial_{z} B_{h}\right)
\end{aligned}
$$

Using the expansion (3.24)-(3.25) and (3.34)-(3.35), we have that

$$
\partial_{t} \omega_{h}+\overline{U_{h}} \cdot \nabla \omega_{h}-\nabla \cdot\left[(1+z-\beta b) \overline{U_{h}}\right] \partial_{z} \omega_{h}=\omega_{h} \cdot \nabla \overline{U_{h}}+\omega_{v} \partial_{z} U_{s h}^{\star}+\mathcal{O}(\sqrt{\mu}) .
$$

Using $\omega_{h}^{\perp}=-\partial_{z} U_{s h}^{\star}, j_{h}^{\perp}=-\partial_{z} B_{s h}^{\star}$ and the fact that $\omega_{v}=\nabla^{\perp} \cdot \overline{U_{h}}+\mathcal{O}(\sqrt{\mu})$ yields $\partial_{t} \omega_{h}+\overline{U_{h}} \cdot \nabla \omega_{h} \nabla \cdot\left[(1+z-\beta b) \overline{U_{h}}\right] \partial_{z} \omega_{h}=\omega_{h} \cdot \nabla \overline{U_{h}}-\left(\nabla^{\perp} \cdot \overline{U_{h}}\right) \omega_{h}^{\perp}+\mathcal{O}(\sqrt{\mu})$.

Taking the perpendicular operator $\perp$, integrating the equation between $z$ and $\epsilon \xi$ and using boundary condition $\partial_{t} \xi+\nabla \cdot Q=0$ at the surface we have that

$$
\partial_{t} U_{s h}+\overline{U_{h}} \cdot \nabla U_{s h}+U_{s h} \cdot \nabla \overline{U_{h}}+\left[\nabla \cdot\left((1+z-\beta b) \overline{U_{h}}\right) \omega_{h}^{\perp}\right]=\mathcal{O}(\sqrt{\mu}) .
$$

Applying operator $\frac{1}{h} \int_{-1+\beta b}^{\xi}$ to (4.12) and subtracting the resulting equation from (4.12), we infer that

$$
\partial_{t} U_{s h}^{\star}+\overline{U_{h}} \cdot \nabla U_{s h}^{\star}+U_{s h}^{\star} \cdot \nabla \overline{U_{h}}-\left[\nabla \cdot\left((1+z-\beta b) \overline{U_{h}}\right) \partial_{z} U_{s h}^{\star}\right]=\mathcal{O}(\sqrt{\mu}) .
$$

Remark 6 Notice that we are working in the particular case of weak magnetic fields (cf. Sect. 3.1) where the plasma- $\beta$ parameter is $\mathcal{O}(1 / \mu)$. Therefore, the evolution of the shear quantity (4.13) coincides with the equation (2.32) in [7] for water waves. For stronger magnetic fields (i.e. plasma- $\beta$ of order $\mathcal{O}(1)$ ), equation (4.13) would contain contributions of the magnetic field, however we would not be able to close the system.

In a similar way, the horizontal component of the current density equation (4.8), is given by

$$
\begin{aligned}
\partial_{t} j_{h} & +U_{h} \cdot \nabla j_{h}+U_{v} \partial_{z} j_{h}-\sqrt{\mu} B_{h} \cdot \nabla \omega_{h}-B_{v} \partial_{z} \omega_{h} \\
= & j_{h} \cdot \nabla U_{h}+\frac{1}{\sqrt{\mu}} j_{v} \partial_{z} U_{h} \\
& -\sqrt{\mu} \omega_{h} \cdot \nabla B_{h}-\omega_{v} \partial_{z} B_{h} \\
& \left.-\left(\nabla^{\perp} B_{h} \cdot \partial_{z} U_{h}-\nabla^{\perp} U_{h} \cdot \partial_{z} B_{h}\right)+\sqrt{\mu}\left(\nabla^{\perp} B_{v} \cdot \partial_{z} U_{v}-\nabla^{\perp} U_{v} \cdot \partial_{z} B_{v}\right)\right)
\end{aligned}
$$


Plugging in the asymptotic expansion (3.24)-(3.25) and (3.34)-(3.35) and dropping terms of order $\mathcal{O}(\sqrt{\mu})$,

$$
\begin{aligned}
& \partial_{t} j_{h}+\overline{U_{h}} \cdot \nabla j_{h}-\nabla \cdot\left[(1+z-\beta b) \overline{U_{h}}\right] \partial_{z} j_{h}-j_{h}^{\mu} \cdot \nabla \overline{U_{h}} \\
& \quad=-\left(\nabla^{\perp} \cdot \overline{U_{h}}\right) \partial_{z} B_{s h}^{\star}+2 \nabla^{\perp} \overline{U_{h}} \cdot \partial_{z} B_{s h}^{\star}+\mathcal{O}(\sqrt{\mu}) .
\end{aligned}
$$

Taking the perpendicular operator $\perp$, integrating in $z$ and using boundary condition $B_{\left.\right|_{z=\xi}} \cdot N=0$ we have that

$$
\begin{aligned}
\partial_{t} & B_{s h}+\overline{U_{h}} \cdot \nabla B_{s h}-\nabla \cdot\left(\overline{U_{h}}(1+z-\beta b)\right) \partial_{z} B_{s h}+\left(\nabla \cdot \overline{U_{h}}\right) B_{s h} \\
= & -\left(B_{s h}^{\perp} \cdot \nabla\right) \overline{U_{h}}+\nabla^{\perp} \cdot \overline{U_{h}} B_{s h}^{\perp} \\
& -2 \nabla^{\perp} \overline{U_{h}}{ }^{\perp} \cdot B_{s h}+\mathcal{O}(\sqrt{\mu}) .
\end{aligned}
$$

Noticing that $\nabla^{\perp}{\overline{U_{h}}}^{\perp}: B_{s h}=\left(B_{s h} \cdot \nabla^{\perp}\right){\overline{U_{h}}}^{\perp}$ and using the vectorial identity

$$
(\nabla \cdot F) G+\left(G^{\perp} \cdot \nabla\right) F^{\perp}+\left(\nabla^{\perp} \cdot F\right) G^{\perp}=(G \cdot \nabla) A
$$

with $F=\overline{U_{h}}$ and $G=B_{s h}$ we have that

$$
\begin{aligned}
(\nabla & \left.\cdot \overline{U_{h}}\right) B_{s h}+\left(B_{s h}^{\perp} \cdot \nabla\right){\overline{U_{h}}}^{\perp}-\left(\nabla^{\perp} \cdot \overline{U_{h}}\right) B_{s h}^{\perp}+2\left(B_{s h} \cdot \nabla^{\perp}\right){\overline{U_{h}}}^{\perp} \\
= & \left(B_{s h} \cdot \nabla\right) \overline{U_{h}}+2\left(B_{s h} \cdot \nabla^{\perp}\right) \overline{U_{h}} \perp \\
& -2\left(\nabla^{\perp} \cdot \overline{U_{h}}\right) B_{s h}^{\perp} \\
= & \left(B_{s h} \cdot \nabla\right) \overline{U_{h}}-2\left(\nabla^{\perp} \overline{U_{h}}\right)^{t} \cdot B_{s h}^{\perp} .
\end{aligned}
$$

Hence, the equation can be rewritten as follows:

$$
\begin{aligned}
& \partial_{t} B_{s h}+\overline{U_{h}} \cdot \nabla B_{s h}+\left(B_{s h} \cdot \nabla\right) \overline{U_{h}}-\nabla \cdot\left(\overline{U_{h}}(1+z-\beta b)\right) \partial_{z} B_{s h} \\
& \quad-2\left(\nabla^{\perp} \overline{U_{h}}\right)^{t} \cdot B_{s h}^{\perp}=\mathcal{O}(\sqrt{\mu}) .
\end{aligned}
$$

Taking the average to (4.17) and subtracting it from (4.17),

$$
\begin{aligned}
& \partial_{t} B_{s h}^{\star}+\overline{U_{h}} \cdot \nabla B_{s h}^{\star}+\left(B_{s h}^{\star} \cdot \nabla\right) \overline{U_{h}}-\nabla \cdot\left(\overline{U_{h}}(1+z-\beta b)\right) \partial_{z} B_{s h}^{\star} \\
& \quad-2\left(\nabla^{\perp} \overline{U_{h}}\right)^{t} \cdot B_{s h}^{\star}{ }^{\perp}=\mathcal{O}(\sqrt{\mu}) .
\end{aligned}
$$

\subsubsection{Closure Equation for $\mathcal{R}, \mathcal{R}_{b}$}

Let us first derive an evolution equation for tensor $\mathcal{R}$. Recall that the evolution equation for the shear velocity $U_{s h}^{\star}$ obtained in (4.13)

$$
\partial_{t} U_{s h}^{\star}+\overline{U_{h}} \cdot \nabla U_{s h}^{\star}+U_{s h}^{\star} \cdot \nabla \overline{U_{h}}-\left[\nabla \cdot\left((1+z-\beta b) \overline{U_{h}}\right) \partial_{z} U_{s h}^{\star}\right]=\mathcal{O}(\sqrt{\mu}) .
$$


Therefore, taking the time derivative on the tensors $\mathcal{R}$, we have that

$$
\begin{aligned}
\partial_{t} \mathcal{R} & =\int_{-1+\beta b}^{\xi} \partial_{t}\left(U_{s h}^{\star} \otimes U_{s h}^{\star}\right)+\partial_{t} \xi\left(U_{s h}^{\star} \otimes U_{s h}^{\star}\right)_{z=\xi} \\
& =\sum_{i=1}^{3} J_{i}+\partial_{t} \xi\left(U_{s h}^{\star} \otimes U_{s h}^{\star}\right)_{\left.\right|_{z=\xi}},
\end{aligned}
$$

where

$$
\begin{aligned}
J_{1} & =-\int_{-1+\beta b}^{\xi} \overline{U_{h}} \cdot \nabla U_{s h}^{\star} \otimes U_{s h}^{\star}+U_{s h}^{\star} \otimes \overline{U_{h}} \cdot \nabla U_{s h}^{\star} \\
& =-\overline{U_{h}} \cdot \nabla \mathcal{R}-\overline{U_{h}} \cdot \nabla(\beta b)\left(U_{s h}^{\star} \otimes U_{s h}^{\star}\right)_{\left.\right|_{z=-1+\beta b}}+\overline{U_{h}} \cdot \nabla \xi\left(U_{s h}^{\star} \otimes U_{s h}^{\star}\right)_{\left.\right|_{z=\xi}}, \\
J_{2} & =-\int_{-1+\beta b}^{\xi}\left(U_{s h}^{\star} \cdot \nabla\right) \overline{U_{h}} \otimes U_{s h}^{\star}+U_{s h}^{\star} \otimes\left(U_{s h}^{\star} \cdot \nabla\right) \overline{U_{h}}=-\nabla{\overline{U_{h}}}^{t} \cdot \mathcal{R}-\mathcal{R} \cdot \nabla \overline{U_{h}},
\end{aligned}
$$

and

$$
\begin{aligned}
J_{3}= & \int_{-1+\beta b}^{\xi}\left[\nabla \cdot\left((1+z-\beta b) \overline{U_{h}}\right) \partial_{z} U_{s h}^{\star}\right] \otimes U_{s h}^{\star}+U_{s h}^{\star} \otimes\left[\nabla \cdot\left((1+z-\beta b) \overline{U_{h}}\right) \partial_{z} U_{s h}^{\star}\right] \\
& \times \int_{-1+\beta b}^{\xi} \nabla \cdot\left((1+z-\beta b) \overline{U_{h}}\right)\left(\partial_{z} U_{s h}^{\star} \otimes U_{s h}^{\star}+U_{s h}^{\star} \otimes \partial_{z} U_{s h}^{\star}\right) \\
= & h\left(U_{s h}^{\star} \otimes U_{s h}^{\star}\right)_{\left.\right|_{z=\xi}}-\left(\nabla \cdot \overline{U_{h}}\right) \mathcal{R}+\nabla(\beta b) \cdot \overline{U_{h}}\left(U_{s h}^{\star} \otimes U_{s h}^{\star}\right)_{\left.\right|_{z=-1+\beta b}} \\
& -\nabla(\beta b) \cdot \overline{U_{h}}\left(U_{s h}^{\star} \otimes U_{s h}^{\star}\right)_{\left.\right|_{z=\xi}}
\end{aligned}
$$

.Using the fact that $\partial_{t} h+\nabla \cdot\left(h \overline{U_{h}}\right)=0$, we obtain that

$$
\partial_{t} \mathcal{R}+\overline{U_{h}} \cdot \nabla \mathcal{R}+\left(\nabla \cdot \overline{U_{h}}\right) \mathcal{R}+\nabla{\overline{U_{h}}}^{t} \mathcal{R}+\mathcal{R} \cdot \nabla \overline{U_{h}}=0
$$

Mimicking the computations but for the magnetic shear equation for $B_{s h}^{\star}$ given by

$$
\begin{aligned}
& \partial_{t} B_{s h}^{\star}+\overline{U_{h}} \cdot \nabla B_{s h}^{\star}+\left(B_{s h}^{\star} \cdot \nabla\right) \overline{U_{h}}-\nabla \cdot\left(\overline{U_{h}}(1+z-\beta b)\right) \partial_{z} B_{s h}^{\star} \\
& \quad-2\left(\nabla^{\perp} \overline{U_{h}}\right)^{t} \cdot B_{s h}^{\star} \perp=\mathcal{O}(\sqrt{\mu}),
\end{aligned}
$$

we arrive at

$\partial_{t} \mathcal{R}_{b}+\overline{U_{h}} \cdot \nabla \mathcal{R}_{b}+\left(\nabla \cdot \overline{U_{h}}\right) \mathcal{R}_{b}+\nabla{\overline{U_{h}}}^{t} \cdot \mathcal{R}_{b}+\mathcal{R}_{b} \cdot \nabla \overline{U_{h}}-\left(\nabla^{\perp} \overline{U_{h}}\right)^{t} \cdot \mathcal{R}_{b}^{S}=0$,

where

$$
\mathcal{R}_{b}^{S}=\int_{-1+\beta b}^{\xi} B_{s h}^{\star}{ }^{\perp} \otimes B_{s h}^{\star}+B_{s h}^{\star} \otimes B_{s h}^{\star}{ }^{\perp} \mathrm{d} z .
$$




\subsection{Full 2D Magnetic Green-Naghdi Equation with Shearing}

We can now express the two-dimensional magnetic Green-Naghdi equation with shearing, dropping $\mathcal{O}\left(\mu^{3 / 2}\right)$ terms by

$$
\left\{\begin{array}{l}
\partial_{t} \xi+\nabla \cdot\left(h \overline{U_{h}}\right)=0, \\
\left.(1+\mu \mathfrak{F})\left(\partial_{t} \overline{U_{h}}+\overline{U_{h}} \cdot \nabla \overline{U_{h}}\right)+\nabla \xi+\mu \mathfrak{D} \overline{\left(\overline{U_{h}}\right.}\right)+\mu \frac{1}{h} \nabla \cdot \mathcal{R}=\mu \overline{B_{h}} \cdot \nabla \overline{B_{h}}+\mu \frac{1}{h} \nabla \cdot \mathcal{R}_{b} \\
\partial_{t} \overline{B_{h}}+\overline{U_{h}} \cdot \nabla \overline{B_{h}}=\overline{B_{h}} \cdot \nabla \overline{U_{h}} \\
\partial_{t} \mathcal{R}+\overline{U_{h}} \cdot \nabla \mathcal{R}+\left(\nabla \cdot \overline{U_{h}}\right) \mathcal{R}+\nabla \overline{U_{h}}{ }^{t} \cdot \mathcal{R}+\mathcal{R} \cdot \nabla \overline{U_{h}}=0 . \\
\partial_{t} \mathcal{R}_{b}+\overline{U_{h}} \cdot \nabla \mathcal{R}_{b}+\left(\nabla \cdot \overline{U_{h}}\right) \mathcal{R}_{b}+\nabla{\overline{U_{h}}}^{t} \cdot \mathcal{R}_{b}+\mathcal{R}_{b} \cdot \nabla \overline{U_{h}}-\left(\nabla^{\perp} \overline{U_{h}}\right)^{t} \cdot \mathcal{R}_{b}^{S}=0,
\end{array}\right.
$$

where the linear operator $\mathfrak{F}[h, b]$ and the quadratic form $\mathfrak{D}[h, b](\cdot)$ are defined for all smooth enough $\mathbb{R}^{2}$ vector valued functions $\mathrm{W}$ by

$$
\begin{aligned}
& \mathfrak{F}[h, b] W=\mathfrak{R}_{1}[h, b](\nabla \cdot W)+\beta \mathfrak{R}_{2}[h, b](\nabla b \cdot W) \\
& \mathfrak{D}[h, b](W)=-2 \mathfrak{R}_{1}\left(\partial_{x} W \cdot \partial_{y} W^{\perp}+(\nabla \cdot W)^{2}\right)+\beta \mathfrak{R}_{2}(W \cdot(W \cdot \nabla) \nabla b),
\end{aligned}
$$

with, for all smooth enough scalar-valued functions $f$

$$
\mathfrak{R}_{1}[h, b] f=-\frac{1}{3 h} \nabla\left(h^{3} f\right)-\beta \frac{h}{2} f \nabla b, \quad \mathfrak{R}_{2}[h, b] f=\frac{1}{2 h} \nabla\left(h^{2} f\right)+\beta f \nabla b,
$$

while the tensors $\mathcal{R}, \mathcal{R}_{b}$ and $\mathcal{R}_{b}^{S}$ stand for

$$
\mathcal{R}=\int_{-1+\beta b}^{\xi} U_{s h}^{\star} \otimes U_{s h}^{\star} \mathrm{d} z, \quad \mathcal{R}_{b}=\int_{-1+\beta b}^{\xi} B_{s h}^{\star} \otimes B_{s h}^{\star} \mathrm{d} z
$$

and

$\mathcal{R}_{b}^{S}=\int_{-1+\beta b}^{\xi} B_{s h}^{\star} \perp B_{s h}^{\star}+B_{s h}^{\star} \otimes B_{s h}^{\star}{ }^{\perp} \mathrm{d} z=M \mathcal{R}_{b}+\mathcal{R}_{b} M^{t}, \quad$ with $M=\left(\begin{array}{cc}0 & -1 \\ 1 & 0\end{array}\right)$.

Remark 7 If we compare the equations with the one derived for the rotational waterwaves in [7], we observe two new phenomena. We have a self interaction of the magnetic shear $B_{s h}^{\star}$ coded in $\mathcal{R}_{b}$ and also an evolution equation for the magnetic field $B_{h}$. Similarly, the magnetic Green-Naghdi equations with shearing introduce several novelties in the system compared to the dispersive equations introduced in [14]. Although we have done the assumptions on the weak magnetic pressure and, therefore, the contributions of the magnetic fields coming from the non-hydrostatic pressure are neglected, we have taken into account the shearing effects as well as the bottom topography variations.

Remark 8 In this remark we will show that if the constraint $\nabla \cdot\left(h \overline{B_{h}}\right)=0$ is imposed on the initial datum at $t=0$ then $\nabla \cdot\left(h \overline{B_{h}}\right)=0$ for later times $t>0$. Indeed, the first and third equation in (4.19) yields 


$$
\begin{aligned}
\partial_{t}\left(h \overline{B_{h}}\right) & =h \partial_{t} \overline{B_{h}}+\overline{B_{h}} \partial_{t} h \\
& =-h \overline{U_{h}} \cdot \nabla \overline{B_{h}}+h \overline{B_{h}} \cdot \nabla \overline{U_{h}}-\overline{B_{h}} \nabla \cdot\left(h \overline{U_{h}}\right) \\
& =-h \overline{U_{h}} \cdot \nabla \overline{B_{h}}+h \overline{B_{h}} \cdot \nabla \overline{U_{h}}-\overline{B_{h}}\left(\overline{U_{h}} \cdot \nabla h\right)-h \overline{B_{h}}\left(\nabla \cdot U_{h}\right),
\end{aligned}
$$

where in the last line we used that $\nabla \cdot\left(h \overline{U_{h}}\right)=\overline{U_{h}} \cdot \nabla h+h\left(\nabla \cdot \overline{U_{h}}\right)$. Moreover, a straightforward computation shows that

$$
-h \overline{U_{h}} \cdot \nabla \overline{B_{h}}-\overline{B_{h}}\left(\overline{U_{h}} \cdot \nabla h\right)=-\overline{U_{h}} \cdot \nabla\left(h \overline{B_{h}}\right)
$$

and hence

$$
\partial_{t}\left(h \overline{B_{h}}\right)=h \overline{B_{h}} \cdot \nabla \overline{U_{h}}-\overline{U_{h}} \cdot \nabla\left(h \overline{B_{h}}\right)-h \overline{B_{h}}\left(\nabla \cdot U_{h}\right) .
$$

Summing up

$$
\left(\partial_{t}+\overline{U_{h}} \cdot \nabla\right)\left(\nabla \cdot\left(h \overline{B_{h}}\right)\right)=-\left(\nabla \cdot\left(h \overline{B_{h}}\right)\right)\left(\nabla \cdot \overline{U_{h}}\right) .
$$

Therefore we deduce that the solenoidal constraint $\nabla \cdot\left(h \overline{B_{h}}\right)=0$ holds for all $t>0$ if $\nabla \cdot\left(h \overline{B_{h}}\right)=0$ at $t=0$.

Remark 9 In contrast to the case of the Green-Naghdi equations with vorticity for water waves [7] (where the magnetic field is absent), it is not a priori clear whether we can derive a local equation for the conservation of energy, due to the last term appearing in the evolution equation for $\mathcal{R}_{b}$ in (4.19).

\subsubsection{Dispersive Effects of the 2D Magnetic Green-Naghdi Equation with Shearing}

In this section we study the dispersive effects of the Green-Naghdi equation with shearing (4.19). First, in order to linearize the equations we need to define a basic state. Taking a mean zero velocity fluid $\left.\overline{\left(U_{h}^{0}\right.}=0\right)$, with a flat surface and flat bottom ( $\xi=0, b=0)$, a uniform ambient magnetic field $\overline{B_{h}^{0}}$ and a uniform vorticity shear $\mathcal{R}^{0}$ and magnetic shear $\mathcal{R}_{b}^{0}$, we can write the variables $\xi, \overline{U_{h}}, \overline{B_{h}}, \mathcal{R}, \mathcal{R}_{b}$ as a sum of the basic sates and small perturbed quantities. Therefore, plugging

$$
\left(\xi, \overline{U_{h}}, \overline{B_{h}}, \mathcal{R}, \mathcal{R}_{b}\right)=\left(\xi^{\prime},{\overline{U_{h}}}^{\prime}, \overline{B_{h}^{0}}+{\overline{B_{h}}}^{\prime}, \mathcal{R}^{0}+\mathcal{R}^{\prime}, \mathcal{R}_{b}^{0}+\mathcal{R}_{b}^{\prime}\right)
$$


into (4.19) and neglecting quadratic small quantities one obtains

$$
\left\{\begin{array}{l}
\partial_{t} \xi^{\prime}+\nabla \cdot{\overline{U_{h}}}^{\prime}=0, \\
\partial_{t}{\overline{U_{h}}}^{\prime}-\frac{\mu}{3} \nabla\left(\nabla \cdot \partial_{t}{\overline{U_{h}}}^{\prime}\right)+\nabla \xi^{\prime}+\mu \nabla \cdot \mathcal{R}^{\prime}=\mu\left(\overline{B_{h}^{0}} \cdot \nabla\right){\overline{B_{h}}}^{\prime}+\mu \mathcal{R}_{b}^{\prime}, \\
\partial_{t}{\overline{B_{h}}}^{\prime}=\left(\overline{B_{h}^{0}} \cdot \nabla\right){\overline{U_{h}}}^{\prime}, \\
\partial_{t} \mathcal{R}^{\prime}+{\overline{U_{h}}}^{\prime} \cdot \nabla \mathcal{R}^{0}+\left(\nabla \cdot \overline{U_{h}}\right)^{\prime} \mathcal{R}^{0}+\left(\nabla{\overline{U_{h}}}^{\prime}\right)^{t} \cdot \mathcal{R}^{0}+\mathcal{R}^{0} \cdot \nabla{\overline{U_{h}}}^{\prime}=0, \\
\partial_{t} \mathcal{R}_{b}^{\prime}+{\overline{U_{h}}}^{\prime} \cdot \nabla \mathcal{R}_{b}^{0}+\left(\nabla \cdot \overline{U_{h}}\right) \mathcal{R}_{b}^{0}+\left(\nabla \overline{U_{h}}\right)^{\prime} \cdot \mathcal{R}_{b}^{0}+\mathcal{R}_{b}^{0} \cdot \nabla{\overline{U_{h}}}^{\prime}-\left(\nabla^{\perp}{\overline{U_{h}}}^{\prime}\right)^{t} \cdot \mathcal{R}_{b}^{S, 0}=0 .
\end{array}\right.
$$

Next, we look for linear waves and derive an equation relating the frequency to the wavevector, i.e. the dispersion relation. To that purpose, we seek for solutions of the form

$$
\left(\xi^{\prime},{\overline{U_{h}}}^{\prime}, \overline{B_{h}}, \mathcal{R}^{\prime}, \mathcal{R}_{b}^{\prime}\right)=\left(\xi^{\prime \prime},{\overline{U_{h}}}^{\prime \prime},{\overline{B_{h}}}^{\prime \prime}, \mathcal{R}^{\prime \prime}, \mathcal{R}_{b}^{\prime \prime}\right) e^{i(k \cdot x-\omega t)}
$$

Substituting (4.22) into (4.19) (and omitting the dash notation) we get the following relations:

$$
\left\{\begin{array}{l}
\overline{U_{h}} \cdot k-\xi \omega=0, \\
-\omega \overline{U_{h}}-\frac{\mu}{3} \omega\left(\overline{U_{h}} \cdot k\right) k+\xi k+\mu \mathcal{R} k=\mu\left(\overline{B_{h}^{0}} \cdot k\right) \overline{B_{h}}+\mu \mathcal{R}_{b} k, \\
-\omega \overline{B_{h}}=\left(\overline{B_{h}^{0}} \cdot k\right) \overline{U_{h}}, \\
-\omega \mathcal{R}+\left(\overline{U_{h}} \cdot k\right) \mathcal{R}^{0}+k\left(\overline{U_{h}}\right)^{t} \cdot \mathcal{R}^{0}+\mathcal{R}^{0} \cdot \overline{U_{h}} k^{t}=0, \\
-\omega \mathcal{R}_{b}+\left(\overline{U_{h}} \cdot k\right) \mathcal{R}_{b}^{0}+k\left(\overline{U_{h}}\right)^{t} \cdot \mathcal{R}_{b}^{0}+\mathcal{R}_{b}^{0} \cdot \overline{U_{h}} k^{t}-k^{\perp}\left(\overline{U_{h}}\right)^{t} \cdot \mathcal{R}_{b}^{S, 0}=0,
\end{array}\right.
$$

where

$$
\mathcal{R}_{b}^{S, 0}=M \mathcal{R}_{b}^{0}+\mathcal{R}_{b}^{0} M^{t}, \quad \text { with } M=\left(\begin{array}{cc}
0 & -1 \\
1 & 0
\end{array}\right)
$$

After some manipulations, eliminating the variables $\xi, \overline{B_{h}}, \mathcal{R}, \mathcal{R}_{b}$, we arrive to

$$
\begin{aligned}
& -\omega^{2} \overline{U_{h}}+\left(k \cdot \overline{U_{h}}\right)\left(-\frac{\mu}{3} \omega^{2} k+k\right)+\mu \\
& \left.\quad \times\left[\left(k \cdot \overline{U_{h}}\right) \mathcal{R}_{T}^{0} k+k \overline{U_{h}} t \mathcal{R}_{T}^{0} k+\mathcal{R}_{T}^{0} \overline{U_{h}} k^{t} k+k^{t}{\overline{U_{h}}}^{t} \mathcal{R}_{b}^{S, 0}+\overline{\left(B_{h}^{0}\right.} \cdot k\right)^{2}\right]=0,
\end{aligned}
$$

where $\mathcal{R}_{T}^{0}=\mathcal{R}^{0}-\mathcal{R}_{b}^{0}$. To simplify the computation, we consider the case where the uniform vorticity and magnetic shear matrices are given by

$$
\mathcal{R}^{0}=\left(\begin{array}{cc}
\delta_{1} & 0 \\
0 & \delta_{1}
\end{array}\right), \quad \mathcal{R}_{b}^{0}=\left(\begin{array}{cc}
\delta_{2} & 0 \\
0 & \delta_{2}
\end{array}\right)
$$


for some $\delta_{1}, \delta_{2} \in \mathbb{R}$. Hence, writing equation (4.24) in matrix form and making the determinant of the matrix equal to zero for non-trivial solutions, we obtain the following dispersion relation:

$$
\omega^{2}=\frac{|k|^{2}+\mu\left(\overline{B_{h}^{0}} \cdot k\right)^{2}+3 \mu\left(\delta_{1}-\delta_{2}\right)|k|^{2}}{1+\frac{\mu}{3}|k|^{2}} .
$$

There appear to be magnetically modified gravity waves and Alfvén modes present in the system influenced by the vorticity and magnetic shearing effects encoded in the difference $\delta_{1}-\delta_{2}$.

Remark 10 It should be noted that in the case $\delta_{1}=\delta_{2}=0$, the dispersion relation (4.25) does not coincide with the dispersion relation showed in Dellar [13]. This is due to the weak magnetic pressure assumption we are working with.

\subsection{The 1D Magnetic Green-Naghdi Equation with Shearing}

For the sake of completeness we also derive the equations in the one-dimensional case. We consider the one dimensional velocity and magnetic fields given by

$$
U=\left(\begin{array}{c}
u \\
0 \\
U_{v}
\end{array}\right), \quad B=\left(\begin{array}{c}
\sqrt{\mu} b \\
0 \\
B_{v}
\end{array}\right)
$$

Therefore, the scalar vorticity and magnetic current are given by

$$
\omega=\left(\begin{array}{c}
0 \\
\omega(x, z, t) \\
0
\end{array}\right), \quad j=\left(\begin{array}{c}
0 \\
j(x, z, t) \\
0
\end{array}\right)
$$

Moreover, we will have that $U_{s h}^{\star}=\left(u_{s h}^{\star}, 0\right)^{t}$ and $B_{s h}^{\star}=\left(b_{s h}^{\star}, 0\right)^{t}$, and hence

$$
u_{s h}^{\star}=-\left(\int_{z}^{\xi} \omega(x, z, t) \mathrm{d} z\right)^{\star}, \quad b_{s h}^{\star}=-\left(\int_{z}^{\xi} j(x, z, t) \mathrm{d} z\right)^{\star}
$$

Therefore, straightforward modifications of the two-dimensional case, yield the following one-dimensional magnetic Green-Naghdi equations:

$$
\left\{\begin{array}{l}
\partial_{t} \xi+\partial_{x}(h \bar{u})=0 \\
(1+\mu \mathfrak{F})\left(\partial_{t} \bar{u}+\bar{u} \partial_{x} \bar{u}\right)+\partial_{x} \xi+\mu \mathfrak{D} \bar{u}+\mu \frac{1}{h} \partial_{x} \mathcal{R}=-\mu Q_{b}^{2} \frac{h_{x}}{h^{3}}+\mu \frac{1}{h} \partial_{x} \mathcal{R}_{b} \\
\partial_{t} \mathcal{R}+\bar{u} \partial_{x} \mathcal{R}+3 \partial_{x} \bar{u} \mathcal{R}=0 \\
\partial_{t} \mathcal{R}_{b}+\bar{u} \partial_{x} \mathcal{R}_{b}+3 \partial_{x} \bar{u} \mathcal{R}_{b}=0
\end{array}\right.
$$


where the one-dimensional versions of $\mathfrak{F}, \mathfrak{D}$ are given by

$$
\begin{aligned}
& \mathfrak{F}[h, b] f=-\frac{1}{3 h} \partial_{x}\left(h^{3} \partial_{x} f\right)+\frac{\beta}{2 h}\left(\partial_{x}\left(h^{2} f \partial_{x} b\right)-h^{2} \partial_{x} f \partial_{x} b\right)+\beta^{2} f\left(\partial_{x} b\right)^{2}, \\
& \mathfrak{D}[h, b] f=-\frac{2}{3 h} \partial_{x}\left(h^{3}\left(\partial_{x} f\right)^{2}\right)+\beta h\left(\partial_{x} f\right)^{2} \partial_{x} b+\frac{\beta}{2 h} \partial_{x}\left(h^{2} f^{2} \partial_{x}^{2} b\right)+\beta^{2} f^{2} \partial_{x}^{2} b \partial_{x} b .
\end{aligned}
$$

Recall that the one dimensional tensors $\mathcal{R}$ and $\mathcal{R}_{b}$ are given by

$$
\mathcal{R}=\int_{-1+\beta b}^{\xi}\left|u_{s h}^{\star}\right|^{2} \mathrm{~d} z, \quad \mathcal{R}_{b}=\int_{-1+\beta b}^{\xi}\left|b_{s h}^{\star}\right|^{2} \mathrm{~d} z
$$

Remark 11 We notice that in the one-dimensional version of the magnetic GreenNaghdi equations with shearing the magnetic field $\bar{b}=0$ trivializes, since $\partial_{x}(h \bar{b})=0$ implies that $\bar{b}=\frac{Q_{b}}{h}$ where $Q_{b}$ is a constant.

Remark 12 In the one-dimensional case it is possible to derive from (4.26) an equation for the local conservation of energy (with $\beta=1, \mu=1$ ), namely

$$
\partial_{t}\left(\mathfrak{e}+\mathfrak{e}_{s h}\right)+\partial_{x}\left(\mathcal{F}+\mathcal{F}_{s h}\right)=0
$$

where the total energy is the sum of is the energy associated to the irrotational frame $\mathfrak{e}$ and a shearing energy $\mathfrak{e}_{S}$

$\mathfrak{e}=\frac{1}{2} \xi^{2}+\frac{1}{2} h|\bar{u}|^{2}+\frac{Q_{b}^{2}}{2 h}+\frac{1}{2} h\left(\frac{1}{3}\left|h \partial_{x} \bar{u}-\frac{3}{2} \partial_{x} b \bar{u}\right|^{2}+\frac{1}{4}\left|\partial_{x} b \bar{u}\right|^{2}\right), \quad \mathfrak{e}_{s h}=\frac{1}{2}\left(\mathcal{R}-\mathcal{R}_{b}\right)$,

and where the flux is given by

$$
\begin{aligned}
\mathcal{F}= & \left(\xi h+\frac{1}{2} h|\bar{u}|^{2}-\frac{Q_{b}^{2}}{2 h}+\frac{1}{2} h\left(\frac{1}{3}\left|h \partial_{x} \bar{u}-\frac{3}{2} \partial_{x} b \bar{u}\right|^{2}+\frac{1}{4}\left|\partial_{x} b \bar{u}\right|^{2}\right)\right. \\
& \left.-\frac{1}{3} h^{2}\left(\partial_{t}+\bar{u} \partial_{x}\right)\left(h \partial_{x} \bar{u}\right)+\frac{1}{2} h^{2}\left(\partial_{t}+\bar{u} \partial_{x}\right)\left(\partial_{x} b \bar{u}\right)\right) \bar{u}
\end{aligned}
$$

and

$$
\mathcal{F}_{\text {sh }}=\frac{3}{2}\left(\mathcal{R}-\mathcal{R}_{b}\right) \bar{u}
$$

\section{Conclusion and Future Work}

In this paper, we have derived new shallow water models for the free-surface MHD equation in the presence of vorticity and magnetic currents under a weak magnetic pressure assumption. An essential ingredient of the model is that the resulting equations are $d$-dimensional which avoid the need to solve the $(d+1)$-dimensional nature of the 
vorticity-current equations, reducing the complexity of the system from a mathematical and numerical point of view. The strategy follows closely the ideas developed in [7]. It is shown that the additional terms appearing in the momentum equation due to the presence of the vorticity and current effects satisfy two advection type equations which are coupled to the system. The advected quantities describe the self-interactions of the shear velocity and magnetic shear induced by the vorticity-current system.

Different techniques were developed to perform numerical simulations for the non-linear shallow MHD equation first derived in [17], for example, the constrained transport approach [12,37], projection method [36] or central upwind scheme $[25,48]$. Therefore, a natural perspective is to take into account the vorticity-current effects in the numerical simulations, allowing the modeling of underlying currents in plasmas or sheared plasmas, using the models derived in this article. Another future research direction, is related to the well-posedness in sufficient regular spaces of the 1D or 2D magnetic Green-Naghdi equation with shearing, which to the best of authors' knowledge, has not been done even in the case of the Green-Naghdi equation with vorticity without magnetic field interactions. In addition, another interesting directions would be extending the precision of the models derived in this article to $\mathcal{O}\left(\mu^{2}\right)$ without any assumption on the amplitude parameter.

Acknowledgements The author is indebted to the anonymous reviewers for providing insightful comments and suggestions which have improve significantly the manuscript. The author is also grateful to Ángel Castro for many useful discussions, supportive observations and encouragement in the course of this work. He also acknowledges helpful conversations with Daniel Faraco and David Lannes.

Funding Open Access funding enabled and organized by Projekt DEAL. The author has been supported by the ICMAT Severo Ochoa project SEV-2015-0554 grant, MTM2017-85934-C3-2-P, ERC grant 834728 Quamap and the Alexander von Humboldt Foundation.

Open Access This article is licensed under a Creative Commons Attribution 4.0 International License, which permits use, sharing, adaptation, distribution and reproduction in any medium or format, as long as you give appropriate credit to the original author(s) and the source, provide a link to the Creative Commons licence, and indicate if changes were made. The images or other third party material in this article are included in the article's Creative Commons licence, unless indicated otherwise in a credit line to the material. If material is not included in the article's Creative Commons licence and your intended use is not permitted by statutory regulation or exceeds the permitted use, you will need to obtain permission directly from the copyright holder. To view a copy of this licence, visit http://creativecommons.org/licenses/by/4.0/.

\section{References}

1. Alvarez-Samaniego, B., Lannes, D.: Large time existence for 3d water-waves and asymptotics. Invent. Math. 171, 485-541 (2008)

2. Alfvén, H.: Existence of electromagnetic-hydrodynamics waves. Nature 150(3), 405-406 (1942)

3. Benzoni-Gavage, S., Serre, D.: Multi-Dimensional Hyperbolic Partial Differential Equations: FirstOrder Systems and Applications. Oxford University Press, Oxford (2007)

4. Bernstein, I.B., Frieman, E.A., Kruskal, M.D., Kulsrud, R.M.: An energy principle for hydromagnetic stability problems. Proc. R. Soc. Lond. Ser. A. 244, 17-40 (1958)

5. Bonneton, P., Chazel, F., Lannes, D., Marche, F., Tissier, M.: A splitting approach for the fully nonlinear and weakly dispersive green-naghdi model. J. Comput. Phys. 230, 1479-1498 (2011)

6. Castro, A., Lannes, D.: Well-posedness and shallow-water stability for a new Hamiltonian formulation of the water waves equations with vorticity. Indiana Univ. Math. J. 64, 1169-1270 (2015) 
7. Castro, A., Lannes, D.: Fully nonlinear long-wave models in the presence of vorticity. J. Fluid Mech. 759, 642-675 (2014)

8. Chen, P., Ding, S.: Inviscid Limit for the Free-Boundary problems of MHD Equations with or without Surface Tension (2019). arXiv:1905.13047

9. Cienfuegos, R., Bartélemy, E., Bonneton, P.: A fourth-order compact nite volume scheme for fully nonlinear and weakly dispersive boussinesq-type equations. Part I: Model development and analysis. Int. J. Numer. Methods Fluids 56, 1217-1253 (2006)

10. Craig, W., Sulem, C.: Numerical simulation of gravity waves. J. Comput. Phys. 108, 73-83 (1993)

11. Da Sterck, H.: Hyperbolic theory of the shallow water magnetohydrodynamics equations. Phys. Plasmas 8, 3293-3304 (2001)

12. DeSterck, H.: Multi-dimensional upwind constrained transport on unstructured grids for shallow water magnetohydrodynamics. AIAA Paper 2001-2623 (2001)

13. Dellar, P.J.: Hamiltonian and symmetric hyperbolic structures of shallow water magnetohydrodynamics. Phys. Plasmas 9, 1130-1136 (2002)

14. Dellar, P.J.: Dispersive shallow water magnetohydrodynamics. Phys. Plasmas 10, 581-590 (2003)

15. Gavrilyuk, S., Gouin, H.: Geometric evolution of the Reynolds stress tensor. Int. J. Eng. Sci. 59, 65-73 (2012)

16. Gilbarg, D., Trudinger, N.S.: Elliptic Partial Differential Equations of Second Order. Springer, Berlin (1983)

17. Gilman, P.A.: Magnetohydrodynamic shallow water equations for the solar tachocline. Astrophys. J. Lett. 544, 1 (2000)

18. Green, A.E., Naghdi, P.M.: A derivation of equations for wave propagation in water of variable depth. J. Fluid Mech. 78, 237-246 (1976)

19. Goedbloed, H.P., Poedts, S.: Principles in Magnetohydrodynamics with Applications to Laboratory and Astrophysical Plasmas. Cambridge University Press, New York (2004)

20. Goedbloed, H.P., Poedts, S.: Advanced Magnetohydrodynamics with Applications to Laboratory and Astrophysical Plasmas. Cambridge University Press, New York (2010)

21. Gu, X., Wang, Y.: On the construction of solutions to the free-surface incompressible ideal magnetohydrodynamic equations. J. Math. Pures Appl. 128, 1-41 (2019)

22. Gu, X.: Well-posedness of axially symmetric incompressible ideal magnetohydrodynamic equations with vacuum under the non-collinearity condition. Commun. Pure. Appl. Anal. 18, 569-602 (2019)

23. Hughes, D., Rosner, R., Weiss, N.: The Solar Tachocline. Springer, New York (2007)

24. Hunter, S.: Waves in Shallow Water Magnetohydrodynamics PhD thesis, University of Leeds, (2013)

25. Kurganov, A., Tadmor, E.: New high-resolution central schemes for nonlinear conservation laws and convection diffusion equations. J. Comput. Phys. 160, 241-282 (2000)

26. Lannes, D.: The Water Waves Problem: Mathematical Analysis and Asymptotics, vol. 188, Mathematical Surveys and Monographs. AMS, Providence (2013)

27. Lannes, D.: Modeling shallow water waves. Nonlinearity 33, 5 (2020)

28. Mak, J., Griffiths, S.D., Hughes, D.W.: Shear flow instabilities in shallow-water magnetohydrodynamics. J. Fluid Mech. 788(10), 767-796 (2016)

29. Mak, J.: Shear instabilities in shallow-water magnetohydrodynamics Ph.D. thesis, University of Leeds (2013)

30. Makarenko, N.: A second long-wave approximation in the Cauchy-Poisson problem. Dyn. Cont. Media 77, 56-72 (1986)

31. Lannes, D., Bonneton, P.: Derivation of asymptotic two-dimensional time-dependent equations for surface water wave propagation. Phys. Fluids 21, 016601 (2009). https://doi.org/10.1063/1.3053183

32. Lannes, D., Marche, F.: A new class of fully nonlinear and weakly dispersive Green-Naghdi models for efficient 2D simulations. J Comput Phys 282, 238-268 (2015). ISSN 0021-9991

33. Ovsjanniko, L.V.: Cauchy problem in a scale of Banach spaces and its application to the shallow water theory justification In: Appl. Meth. Funct. Anal. Probl. Mech. (IUTAM/IMU-Symp., Marseille. Lect. Notes Math., vol. 503, pp. 426-437 (1975)

34. Richard, G.L., Gavrilyuk, S.L.: A new model of roll waves: comparison with Brock's experiments. J. Fluid Mech. 698, 374-405 (2012)

35. Richard, G.L., Gavrilyuk, S.L.: The classical hydraulic jump in a model of shear shallow-water flows. J. Fluid Mech. 725, 492-521 (2013)

36. Rossmanith, J.A.: A wave propagation method with constrained transport for ideal and shallow water magnetohydrodynamics. Ph.D. thesis, University of Washington (2002) 
37. Rossmanith, J.A.: A Constrained Transport Method for the Shallow Water MHD Equations. In: Hou, T.Y., Tadmor, E. (eds.) Hyperbolic Problems: Theory, Numerics, Applications. Springer, Berlin, Heidelberg (2003)

38. Schecter, D.A., Boyd, J.F., Gilman, P.A.: Shallow water magnetohydrodynamic waves in the solar tachocline. Astrophys. J. 551, 185-188 (2001)

39. Serre, F.: Contribution a l'etude des ecoulements permanents et variables dans les canaux. La Houille Blanche 8, 830-872 (1953)

40. Spiegel, E., Zahn, J.P.: The solar tachocline. Astron. Astrophys. 265, 106-114 (1992)

41. Teshukov, V.M.: Gas-dynamic analogy in the theory of stratified liquid flows with a free boundary. Izv. Ross. Akad. Nauk Mekh. Zhidk. Gaza 5, 143-153 (2007)

42. Trakhinin, Y.: Stability of relativistic plasma-vacuum interfaces. J. Hyperbol. Differ. Equations 9(3), 469-509 (2012)

43. Trakhinin, Y.: On well-posedness of the plasma-vacuum interface problem: the case of non-elliptic interface symbol. Commun. Pure. Appl. Anal. 15(4), 1371-1399 (2016)

44. Trakhinin, Y.: Structural stability of shock waves and current-vortex sheets in shallow water magnetohydrodynamics (2019). arXiv:1911.06295

45. Warneforda, E., Dellar, P.: Thermal shallow water models of geostrophic turbulence in Jovian atmospheres. Phys. Fluids 150(3), 405-406 (2014)

46. Zakharov, V.E.: Stability of periodic waves of finite amplitude on the surface of a deep fluid. J. Appl. Mech. Tech. Phys. 9, 190-194 (1968)

47. Zeitlin, V.: Remarks on rotating shallow-water magnetohydrodynamics. Nonlinear Proc. Geophys. 20, 893-898 (2013)

48. Zia, S., Qamar, M.A.S.: Numerical solution of shallow water magnetohydrodynamic equations with non-flat bottom topography. Int. J. Comput. Fluid Dyn. 28(1-2), 56-75 (2014)

49. Cally, P.S.: Three-dimensional magneto-shear instabilities in the solar tachocline. Mon. Not. R. Astron. Soc. 339(4), 957-972 (2003)

Publisher's Note Springer Nature remains neutral with regard to jurisdictional claims in published maps and institutional affiliations. 